\title{
Transverse impedance of tapered transitions with elliptical cross section
}

\author{
B. Podobedov and S. Krinsky \\ Brookhaven National Laboratory, Upton, New York 11973, USA
}

(Received 23 May 2007; published 30 July 2007)

\begin{abstract}
We study the transverse geometric impedance of elliptical cross-section tapers in the low-frequency "inductive regime." We have followed a dual approach: computer simulations have been carried out using the finite element electromagnetic code GDFIDL and analytic results for the dipolar and quadrupolar components of the impedance have been derived extending a perturbation technique introduced by Stupakov. Our work provides new insight into the behavior of the impedance of axially asymmetric tapered structures at low frequency. In particular, we clarify the frequency range characterizing the inductive regime, suggesting new criteria relating the extent of the inductive regime for dipolar and quadrupolar components of the impedance to the dimensions of the minimal cross section of a tapered transition.
\end{abstract}

DOI: 10.1103/PhysRevSTAB.10.074402

PACS numbers: 41.20.Jb, 41.60.- $-\mathrm{m}$

\section{INTRODUCTION}

It is well known that vacuum chamber discontinuities in particle accelerators lead to strong wakefield interactions that can cause collective instabilities and other unwanted effects. Therefore, transitions between segments of the vacuum chamber having different transverse crosssectional geometries must be carefully designed and smoothly tapered. An important practical question is how to estimate the impedance of tapers going from one cross section to another in a given length. In particular, since small-gap undulators are central to the design of modern high-brightness synchrotron light sources, the determination of the transverse impedance of the tapered vacuum chambers for these devices is of paramount importance. One must determine how gradual tapers must be to assure that the desired number of small-gap insertion devices can be installed in the storage ring before the impedance budget is exceeded. Similar issues arise in the design of linear colliders where impedance due to tapered collimators may degrade beam quality.

At present, there is no general analytical formalism to determine the impedance of tapers for realistic vacuum chamber geometries. Computer simulations can be used as a guide, but obtaining reliable results is not always straightforward, especially for very smooth chambers. Here, we are concentrating on the parameter range where tapering is effective in reducing the impedance, which implies that the tapers are very gradual. Furthermore, we are interested in cases of large cross-sectional variation, for example, when the vertical aperture changes by a substantial factor. We concentrate on the transverse impedance of tapered transitions, as the corresponding longitudinal impedance is usually small in comparison with that due to other machine components.

The work in this paper draws upon earlier investigations by Yokoya [1] and Stupakov [2] of the impedance of structures with large but gradual variation of beam pipe dimensions. Yokoya [1] discussed the geometric impedance of a slowly tapered circular chamber. Further understanding was provided by Stupakov [2] who showed that Yokoya's results were valid down to zero frequency. Stupakov [3] then presented an analysis of a flat tapered structure with rectangular cross section, and found that the impedance at low frequency can be significantly larger than that of a chamber with circular cross section.

Yokoya's result for the low-frequency transverse dipolar impedance of an axially symmetric tapered transition is given by [1]

$$
Z_{\perp}^{\text {round }}(k) \cong \frac{-i Z_{0}}{2 \pi} \int_{-\infty}^{\infty} d z \frac{r^{\prime}(z)^{2}}{r(z)^{2}},
$$

where $k$ is the wave number of the perturbing field, $Z_{0}$ is the free space impedance, $r(z)$ is the radius of the tapered chamber, and the prime denotes derivative with respect to the axial coordinate $z$. If the variation of the radius takes place over a characteristic distance $L$, then Yokoya's approximation holds under two conditions: (i) $r_{\mathrm{av}} \ll L$ [4], where $r_{\mathrm{av}}$ is the average value of radius over the variation; and (ii) $k r_{\min } r^{\prime} \ll 1$ [1], where $r_{\min }$ is the minimum radius and $r^{\prime}$ is a characteristic value of the boundary derivative. We have recently extended Stupakov's [2] formalism to include higher-order terms in the perturbation expansion [4], and derived an approximation to the low-frequency impedance valid for all taper lengths. Our work in Ref. [4] provides an interpolation between Yokoya's result [Eq. (1.1)] valid for a slow taper and the impedance of a step transition.

Stupakov [3] ${ }^{1}$ has derived an approximation for the vertical-dipolar impedance of a flat rectangular chamber of constant half-width $w$ and varying half-gap $h(z) \ll w$,

\footnotetext{
${ }^{1}$ It has been recently found that the Ref. [3] result is a factor of 2 too high [5]. In Eq. (1.2) and throughout the paper, we refer to the corrected result.
} 


$$
Z_{D y}^{\mathrm{flat}}(k) \cong \frac{-i Z_{0} w}{4} \int_{-\infty}^{\infty} d z \frac{h^{\prime}(z)^{2}}{h(z)^{3}}
$$

The impedance of the flat chamber [Eq. (1.2)] has a form similar to Eq. (1.1) for a circular chamber, but is larger by a big factor $\sim w / h_{\mathrm{av}}$, where $h_{\mathrm{av}}$ is the average height. This is a strong motivation to go beyond the approximate estimates one would get by replacing the real geometry with an axially symmetric one. The range of validity for Eq. (1.2) is not clearly established. If the variation of the gap takes place over a distance $L$, then we expect that a necessary condition at zero frequency is $h_{\text {av }} \ll w \ll L$. Stupakov [6] suggests that the condition $k w^{2} \ll L$ is also necessary. Our present work suggests that, in order for Eq. (1.2) to hold, it is also necessary that $k w<1$, which is a more restrictive condition for a long taper. We also find that for structures with significant horizontal-to-vertical aspect ratio a related condition, $\sigma_{z} \cong 2 w / \pi$, describes characteristic bunch length value below which the vertical kick factor, $\kappa_{D y}$, transitions from inductive $\left(\kappa_{D y} \sim \sigma_{z}^{-1}\right)$ to intermediate $\left(\kappa_{D y} \sim \sigma_{z}^{-(1 / 2)}\right)$ behavior.

A flat rectangular geometry is not typically chosen for accelerator tapers. Elliptical or rounded-corner rectangular cross sections with aspect ratios of 2-5 are much more common. Apart from practical relevance, elliptical geometry is also convenient for advancing our theoretical understanding, since it accommodates circular as well as nearly flat geometries.

In the present paper we have pursued a dual approach to the study of the impedance of elliptical tapers. Computer simulations have been carried out using the finite element electromagnetic code GDFIDL [7] and analytic perturbation theory results have been derived using the technique introduced by Stupakov [2]. Some of our preliminary results have been briefly described in [8].

In the case of a circular cross section, there is a broad low-frequency inductive regime in which the real part of the impedance vanishes and the imaginary part is slowly varying. For an elliptic structure, we have found that over the full range of aspect ratios [ratio of major $(x)$ to minor $(y)$ axes] the major-axis dipolar impedance, $Z_{D x}$, and the quadrupolar impedance, $Z_{Q}$, exhibit a broad low-frequency inductive regime (up to $k \sim 1 / b_{\min }$, where $b_{\min }$ is the minor-axis of the elliptical chamber at the minimal cross section). However, the low-frequency minor-axis dipole impedance, $Z_{D y}$, behaves in this simple inductive manner over a broad frequency range only when the structure is close to circular in cross section. As the aspect ratio increases, the inductive region becomes confined to lower and lower frequencies and for relatively flat structures it extends roughly up to $k \sim 2 / a_{\min }$, where $a_{\min }$ is the major semiaxis of the elliptical chamber at the minimal cross section. Correspondingly, we find that first-order perturbation theory provides a very accurate description of the lowfrequency behavior of the major-axis and quadrupolar impedances. However, for the minor-axis impedance, the agreement between the first-order perturbation theory and the GDFIDL calculations becomes less precise as the aspect ratio increases. Nevertheless, when in the inductive regime, we can accurately describe all three impedance components by simple analytical formulas, derived in this paper.

The paper is organized as follows: In Sec. II, we present results of GDFIDL calculations of the impedance and wakefield for elliptic tapers, and clarify the frequency range of the inductive regime. In Sec. III, we use the perturbation approach to derive analytic approximations for the impedance at zero frequency. The criterion for the applicability of this method is not obvious. Although it is based on the slow variation of the tapered transition, it is not clear precisely what is the small parameter justifying the neglect of higher-order terms. Therefore, in Sec. IV, we obtain the conditions justifying the perturbation expansion, which are found to be significantly different for the vertical impedance than for the horizontal and quadrupolar when the aspect ratio is high. In Sec. V, comparing perturbation results with GDFIDL calculations, we confirm the analysis of Sec. IV by showing that the lowest-order perturbation method gives very accurate results for the horizontal and quadrupolar impedances for all aspect ratios. The behavior of the vertical-dipole impedance is more complex, and the accuracy of the first-order approximation becomes worse for large aspect ratio. In Sec. VI, we summarize our results and make some concluding remarks. Finally, in the Appendix we provide more details of our GDFIDL calculations.

\section{GDFIDL CALCULATIONS FOR TAPERS IN THE INDUCTIVE REGIME}

\section{A. Preliminaries}

We begin with a qualitative discussion of wakefields and impedances of tapered structures. Our emphasis in this paper is on the short-range transverse wakefields (and consequently broadband transverse impedances), i.e., those that act predominantly within a single bunch. As such, our consideration is inherently bunch length dependent. Since the wakefields and impedances of circular structures are well known [1], our goal is to clarify the new features that arise when the circular symmetry is broken. Of particular interest is the important limit of "flat structures" (here and below this refers to the structures with large aspect-ratio cross sections).

To avoid misunderstanding, let us first review the definition of transverse wakefields and impedance. Consider a point charge $q$ (the drive particle) traveling very close to the speed of light in the $z$-direction through a vacuum enclosure, displaced from the design trajectory by $\vec{r}_{d}=$ $\left(x_{d}, y_{d}\right)$. A unit test charge travels at a distance $s$ behind the first on a trajectory parallel to the $z$-axis but displaced by $\vec{r}_{t}=\left(x_{t}, y_{t}\right)$. The transverse wakefield is given by 


$$
\begin{aligned}
w_{x, y}\left(\vec{r}_{d}, \vec{r}_{t}, s\right)= & \frac{1}{q} \int_{-\infty}^{\infty} d z\left[\vec{E}\left(\vec{r}_{d} ; \vec{r}_{t}, z, t=\frac{z+s}{c}\right)\right. \\
& \left.+c \hat{z} \times \vec{B}\left(\vec{r}_{d} ; \vec{r}_{t}, z, t=\frac{z+s}{c}\right)\right]_{x, y} .
\end{aligned}
$$

The transverse impedance is determined by

$$
Z_{x, y}\left(\vec{r}_{d}, \vec{r}_{t}, k\right)=\frac{-i}{c} \int_{0}^{\infty} d s w_{x, y}\left(\vec{r}_{d}, \vec{r}_{t}, s\right) e^{i k s}
$$

When the vacuum enclosure has reflection symmetry about the $x z$ and the $y z$ planes, the transverse wakefield vanishes for $\vec{r}_{t}=\vec{r}_{d}=0$ and the first terms in a Taylor expansion yield [9] ${ }^{2}$

$$
\begin{aligned}
& w_{x}\left(x_{d}, y_{d}, x_{t}, y_{t}, s\right) \cong w_{D x}(s) x_{d}+w_{Q}(s) x_{t}, \\
& w_{y}\left(x_{d}, y_{d}, x_{t}, y_{t}, s\right) \cong w_{D y}(s) y_{d}-w_{Q}(s) y_{t},
\end{aligned}
$$

where $w_{D}$ is the dipole wakefield and $w_{Q}$ is the quadrupolar wakefield. The dipole and quadrupolar impedances are determined by Fourier transform as in Eq. (2.2):

$$
\begin{aligned}
& Z_{x}\left(x_{d}, y_{d}, x_{t}, y_{t}, k\right) \cong Z_{D x}(k) x_{d}+Z_{Q}(k) x_{t}, \\
& Z_{y}\left(x_{d}, y_{d}, x_{t}, y_{t}, k\right) \cong Z_{D y}(k) y_{d}-Z_{Q}(k) y_{t} .
\end{aligned}
$$

For undulator chambers with small vertical gap, the vertical-dipole impedance is the largest component. However, it may be that the undulator is situated in an insertion having horizontal beta function large compared to the vertical. In this case, the horizontal impedance can be important in determining the instability threshold, since it is the product of the beta function times the transverse impedance which enters the threshold condition (see e.g. [10]).

The quadrupolar impedance vanishes for chambers with circular cross section, but not for axially asymmetric structures. Understanding the quadrupolar impedance is important because: (i) incoherent tune shifts may significantly affect single particle dynamics of a circular machine, for example, by pushing it onto a resonance [11]; (ii) Landau damping due to this tune shift may suppress coherent instabilities [12]; (iii) quadrupolar impedance can drive quadrupole beam breakup instability in linacs [13]; (iv) it is necessary to account for quadrupolar impedance in machine-based impedance measurements [14-16]; (v) separation of the dipolar and quadrupolar impedances is important for bench measurements of impedance using the stretched wire technique [17].

\footnotetext{
${ }^{2}$ This reference deals with the special case of long range resistive wall, where $w_{D}$ and $w_{Q}$ differ only by a numerical factor but have the same $s$-dependence. This is not true in general (see e.g. [18]) and for the geometric taper impedance we consider in this paper.
}

The geometric quadrupolar impedance can be comparable to the quadrupolar component of the resistive wall impedance, first derived in [18]. For example, this may be the case in the light sources that heavily utilize small-gap undulators. ESRF [10] and SOLEIL [19] report that 30\%$50 \%$ of the quadrupolar impedance may come from the geometry and the rest from the resistive wall. As newer light sources utilize an even larger number of small-gap undulators, the effect of the geometric quadrupolar impedance may become even more significant [20].

\section{B. GDFIDL calculations}

We illustrate our discussion with calculations performed using the code GDFIDL for the geometry of Fig. 1, consisting of a uniform pipe with elliptical cross section linearly tapered to another uniform elliptical pipe with a larger cross section; the structure is then continued mirror symmetrically with respect to the middle of the center pipe. In our calculations, we fix the minimum and maximum minor semiaxes to be $b_{\min }=1 \mathrm{~cm}$ and $b_{\max }=4.5 \mathrm{~cm}$ and the tapering occurs over a fixed distance $2 L=20 \mathrm{~cm}$. Dimensions are chosen so that the height variation is large, $b_{\max } / b_{\min }=4.5$, but the tapering in both horizontal and vertical planes is gradual $\left(b_{\max }-b_{\min }\right) / 2 L \ll 1$ and $\left(a_{\max }-a_{\min }\right) / 2 L \ll 1$. The impedance of the axially symmetric limit of this structure is well described by Eq. (1.1), as was confirmed in [4].

The straight pipe between the transitions is chosen long enough to assure that for low frequency, we are in the regime of two noninteracting tapers. In the regime of interest the wakefield and impedance of the convex structure is expected to be the same as that of a corresponding concave structure; a convex geometry (Fig. 1) was chosen to reduce numerical noise. Details of GDFIDL calculations as well as some consistency checks are discussed in the Appendix.

The vertical minor semiaxes $\left(b_{\min }, b_{\max }\right)$ and axial dimensions ( $L$ and nonessential lengths of uniform pipes) shown in Fig. 1 are fixed for all calculations presented below. Both the outer and the inner pipes are then varied from round to approximately flat maintaining the confocal condition between the two, $a_{\min }^{2}-b_{\min }^{2}=a_{\max }^{2}-b_{\max }^{2}$. Qualitative conclusions of this section do not actually require this condition. It is imposed here for convenience

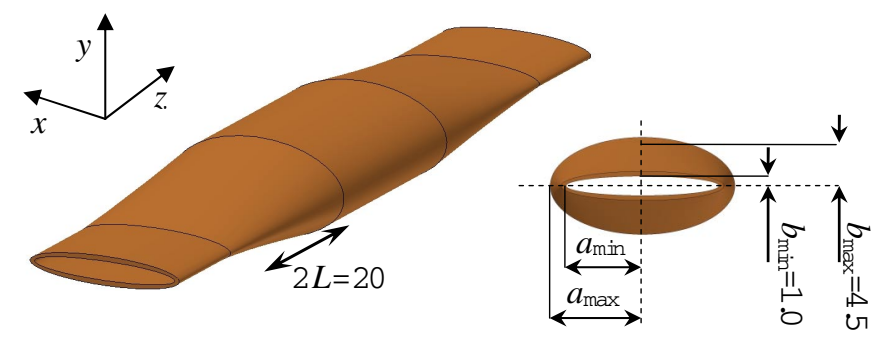

FIG. 1. (Color) Geometry with principal dimensions in $\mathrm{cm}$. 
only because we later derive analytical expressions for the impedance of confocal-elliptical structures. ${ }^{3}$ With the vertical dimensions fixed, the confocal condition connects the aspect ratios of the larger and smaller pipes. For simplicity, we will characterize the degree of flatness of the structure in terms of the horizontal-to-vertical aspect ratio of the smaller cross-section pipe $a_{\min } / b_{\min }$, where the structure is the flattest.

\section{Wake potentials}

The time-domain electromagnetic code GDFIDL calculates the wake potentials due to a Gaussian drive bunch of given length. The wake potentials $(W)$ due to a drive bunch of finite length are related to the wakefields $(w)$ due to a point charge by

$$
W_{x, y}(s)=\int_{-\infty}^{\infty} d s^{\prime} w_{x, y}\left(s-s^{\prime}\right) f\left(s^{\prime}\right)
$$

where $f(z)$ is the unity normalized longitudinal beam density. Similarly, we can define the dipolar and quadrupolar wake potentials by

$$
W_{D x, D y, Q}(s)=\int_{-\infty}^{\infty} d s^{\prime} w_{D x, D y, Q}\left(s-s^{\prime}\right) f\left(s^{\prime}\right)
$$

By choice of appropriate boundary conditions at the symmetry planes of the structure, we can separately calculate the dipolar and quadrupolar wake potentials defined in Eq. (2.8). See the Appendix for more detail. Figures 2-4 present the wake potentials resulting from a drive bunch with rms length of $1 \mathrm{~cm}$.

As we change the geometry from round to almost flat, the wake potentials exhibit rather different behavior. For the horizontal and quadrupolar cases, the wake potentials are approximately proportional to the bunch shape (Figs. 2 and 3), implying purely imaginary impedance that is constant with frequency. In the limit of a round structure, the quadrupolar wake vanishes as expected, while in the opposite limit of a flat structure, the sum of the quadrupolar and horizontal wake potentials vanishes, as it must since there is no total force experienced by a charge displaced horizontally in a flat structure.

For the vertical wake potential the situation is different as indicated in Fig. 4, where we present the wake potentials from GDFIDL for aspect ratios up to 64. The wake potentials for small aspect ratios are roughly Gaussian. However, for aspect ratio greater than $\sim 2$, significant "ringing" appears behind the drive bunch. As the aspect ratio is increased, the wake potential starts widening significantly beyond the drive bunch shape, while its peak height saturates.

\footnotetext{
${ }^{3}$ Note that since we linearly taper both semiaxes, the confocal condition is violated along the taper; however, this was found to have virtually no effect on the results.
}

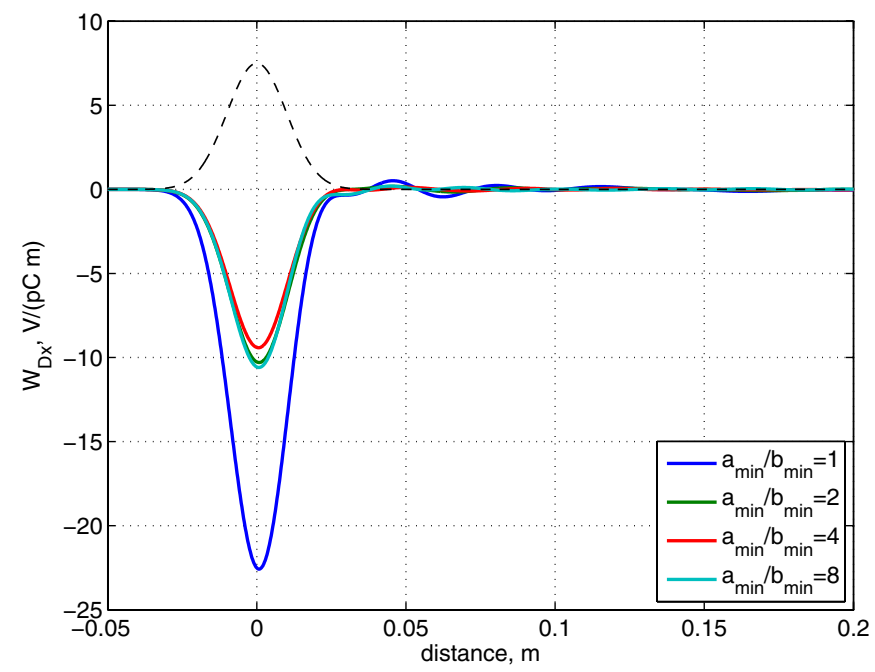

FIG. 2. (Color) Dipolar-horizontal wake potentials (solid line) and beam density (dashed line).

\section{Impedances and the frequency range of the inductive regime}

Insight into the differences in behavior exhibited by the vertical-dipolar impedance on the one hand and the horizontal and quadrupolar impedances on the other, are easier to explain in the frequency domain. The horizontal, quadrupolar, and vertical impedances vs frequency (obtained in GDFIDL from the Fourier transformed wake potentials extending $2 \mathrm{~m}$ behind the $1 \mathrm{~cm}$ rms long driving Gaussian bunch) are presented in Figs. 5-7.

We observe that for every aspect ratio there is a frequency range extending from zero frequency upwards where, to a good accuracy, the real part of impedance vanishes. The imaginary part is essentially constant in this frequency range. Since no real part in the transverse

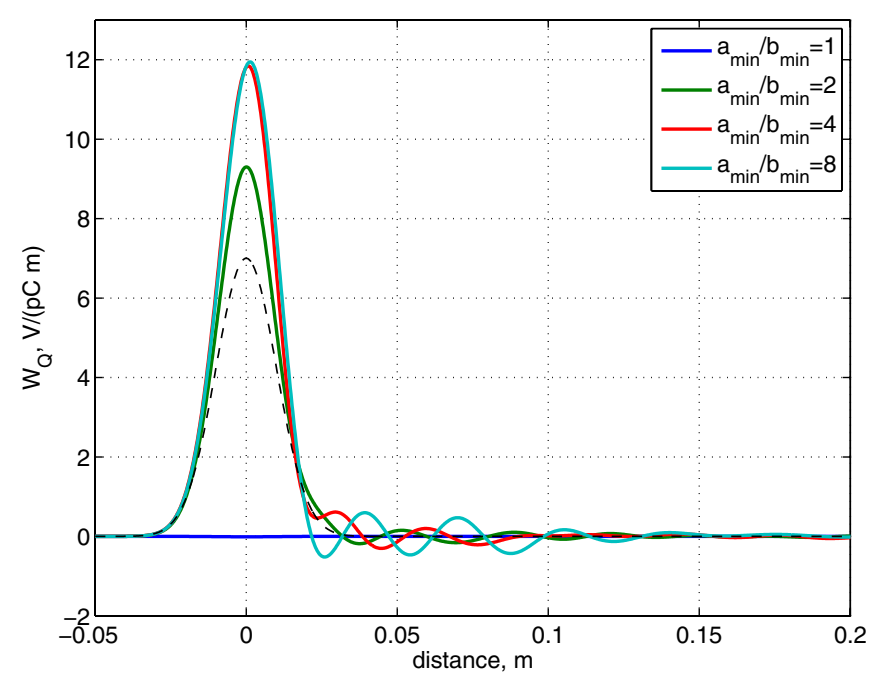

FIG. 3. (Color) Quadrupolar wake potentials (solid line) and beam density (dashed line). 


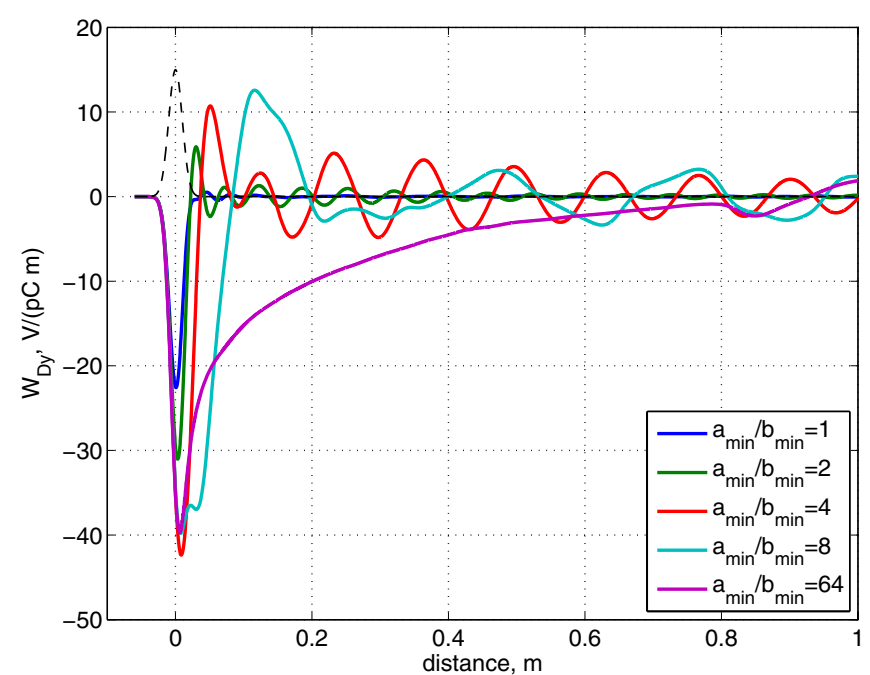

FIG. 4. (Color) Dipolar vertical wake potentials (solid line) and beam density (dashed line).

impedance implies no energy losses, this regime is called "inductive." It is this regime that is the main focus of our paper.

For the quadrupolar and horizontal impedances, the frequency range over which $\operatorname{Re}[Z(\omega)]=0$ and $\operatorname{Im}[Z(\omega)]=$ const does not significantly change as the structure gets flatter. In contrast, for the vertical-dipolar impedance, the frequency range of the inductive regime narrows as $\sim 1 / a_{\min }$ as the structure flattens.

This behavior is easy to understand as a consequence of the fact that wave propagation in a waveguide is only possible at frequencies above the lowest cutoff frequency, which (for a tapered waveguide) is in turn determined by the smallest cross section, see e.g. [21]. Therefore, if a beam is long enough to not effectively excite those frequencies, it does not strongly interact with the waveguide
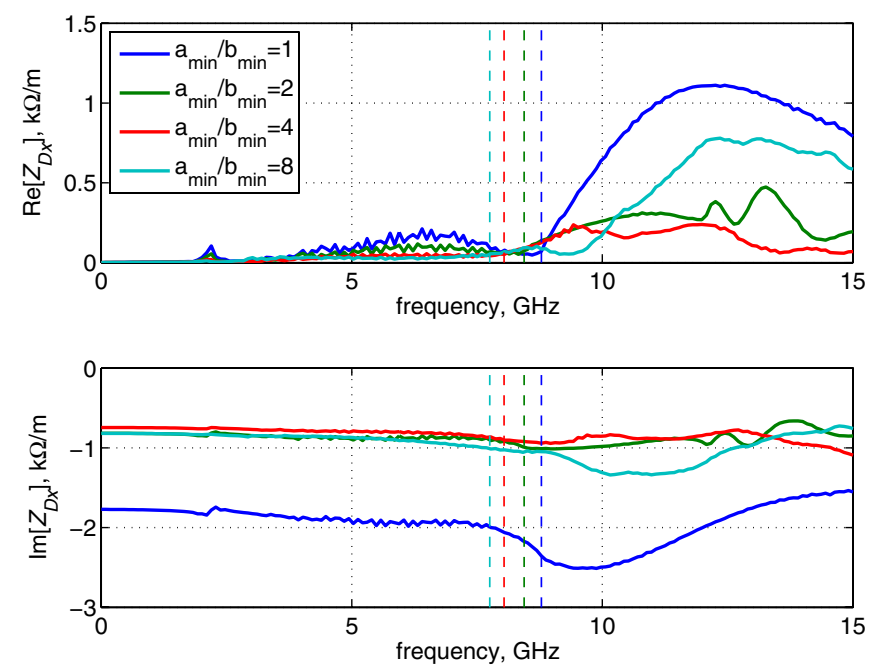

FIG. 5. (Color) Dipolar-horizontal impedance (solid line) and ${ }_{5} \mathrm{H}_{11}$ cutoff frequencies of the structure (dashed line).
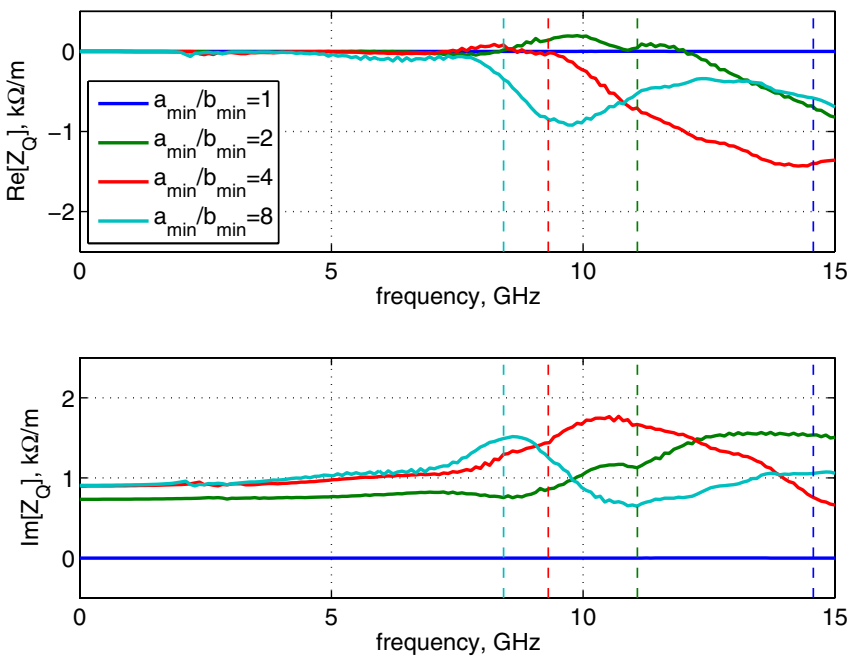

FIG. 6. (Color) Quadrupolar impedance (solid line) and ${ }_{5} \mathrm{H}_{21}$ cutoff frequencies of the structure (dashed line).

modes and cannot lose energy to them. In the context of tapered accelerator structures, where H-type modes are relevant for the transverse impedance, this has been emphasized by Stupakov [6]. Blednykh [22] has further investigated the vertical impedance in asymmetric structures and has demonstrated the existence of narrow-band "trapped" modes in the vicinity of the lowest cutoff frequency. Narrow-band impedance at cutoff is complicated and it essentially depends on the nontapered parts of the structure [22], as well as whether it is concave or convex, and likely many other factors. Here, however, we concentrate on the broadband impedance and inductive regime, so these complexities are not critical. Our conclusion is that the frequency range of the inductive regime extends from zero up to the cutoff frequency of the appropriate mode, which can be identified from the basic considerations to follow.
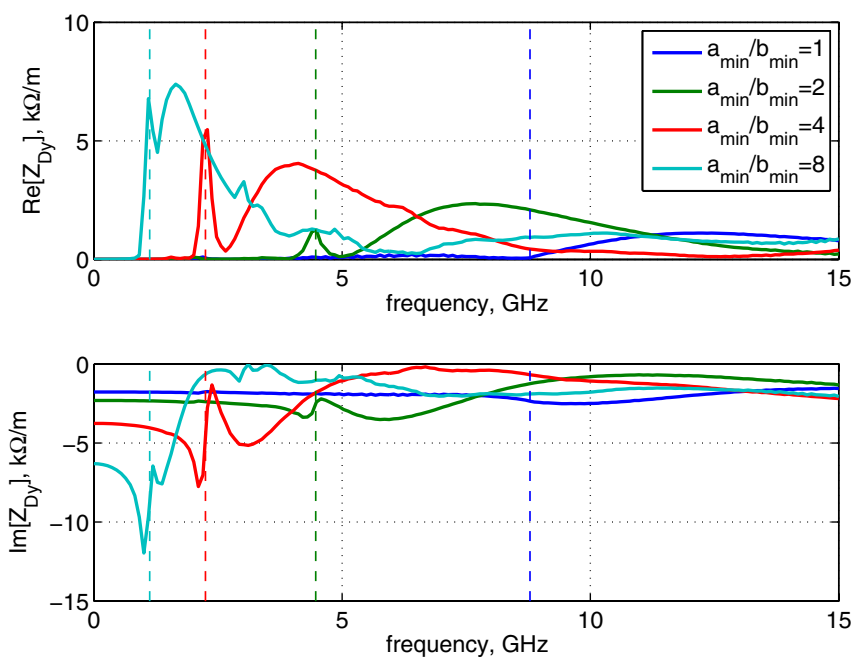

FIG. 7. (Color) Dipolar vertical impedance (solid line) and ${ }_{\mathrm{C}} \mathrm{H}_{11}$ cutoff frequencies of the structure (dashed line). 

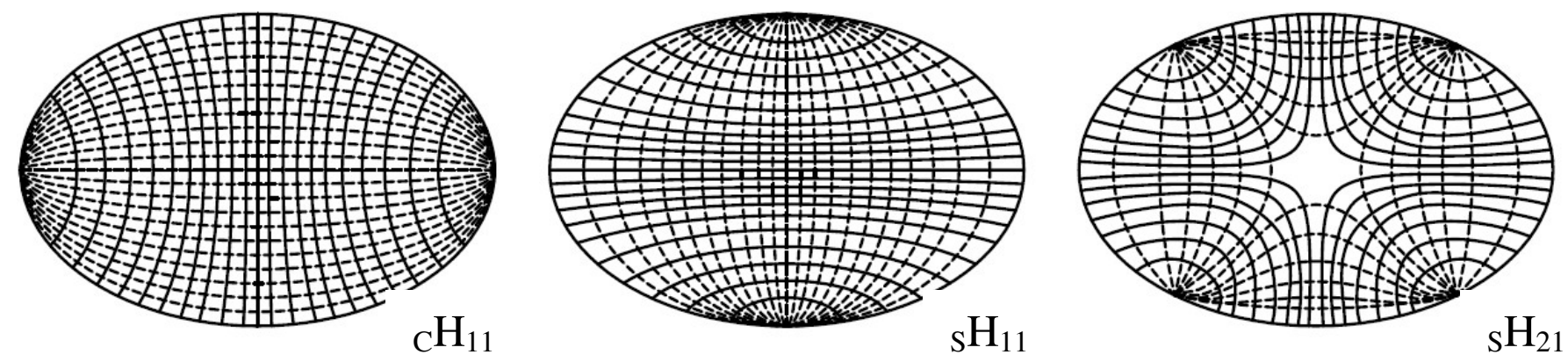

FIG. 8. Field patterns (electric — solid line, magnetic — dashed line) for ${ }_{\mathrm{C}} \mathrm{H}_{11},{ }_{\mathrm{S}} \mathrm{H}_{11}$, and $\mathrm{S}_{\mathrm{S}} \mathrm{H}_{21}$ modes in elliptical waveguide.

To contribute to dipolar or quadrupolar impedance, a mode must have the proper symmetry. Additionally, for the dipolar case, the mode must have an appropriate (horizontal or vertical) electric field direction. These two conditions plus the requirement of the lowest cutoff frequency uniquely define the mode in question. Specifically, the lowest cutoff frequency modes that limit the inductive impedance range are ${ }_{C} \mathrm{H}_{11}$ (vertical), ${ }_{5} \mathrm{H}_{11}$ (horizontal), and ${ }_{5} \mathrm{H}_{21}$ (quadrupolar). Cartoon drawings of EM fields for these modes are shown in Fig. 8 (see e.g. [21]). We have calculated the cutoff frequencies for these modes for our geometry using approximate formulas from [23], and added the resulting values in the dashed line to the impedance Figs. 5-7. Clearly these cutoff frequencies can be taken as approximate upper boundaries of the inductive regime.

For reference purposes we also present a separate figure, Fig. 9, that shows the cutoff wavelength for the aforementioned elliptical waveguide modes $\left({ }_{\mathrm{C}} \mathrm{H}_{11},{ }_{\mathrm{S}} \mathrm{H}_{11}\right.$, and $\left.{ }_{\mathrm{S}} \mathrm{H}_{21}\right)$ as well as the corresponding rectangular waveguide modes $\left(\mathrm{H}_{10}, \mathrm{H}_{01}\right.$, and $\left.\mathrm{H}_{11}\right)$. The rectangular example is convenient

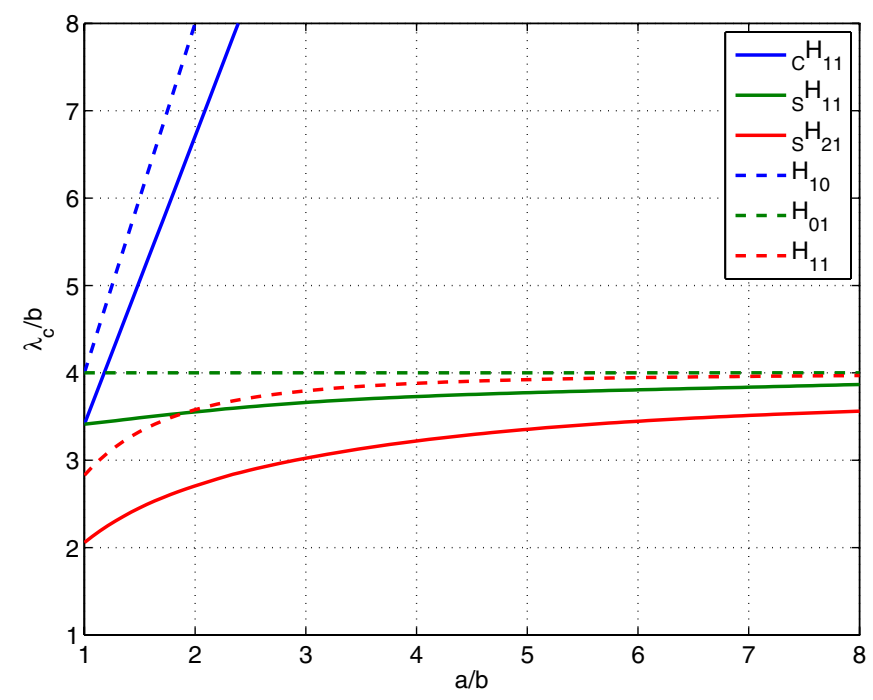

FIG. 9. (Color) Cutoff wavelengths for the three lowest $\mathrm{H}$ modes in elliptical (solid line) and rectangular (dashed line) waveguides. $a$ and $b$ are half-width and half-height in both cases. as it has simple formulas for the cutoff frequencies, specifically

$$
\begin{gathered}
f_{c}^{\mathrm{H}_{10}}=\frac{c}{4 a_{\min }}, \\
f_{c}^{\mathrm{H}_{01}}=\frac{c}{4 b_{\min }}, \\
f_{c}^{\mathrm{H}_{11}}=\frac{c \sqrt{1+b_{\min }^{2} / a_{\min }^{2}}}{4 b_{\min }},
\end{gathered}
$$

where $a_{\min }$ and $b_{\min }$ stand for half-width and half-height of the rectangular cross section.

As expected, the scaling of the cutoff wavelength with the waveguide dimensions for the corresponding modes of the elliptical and rectangular cross sections is quite similar except that elliptical modes are somewhat lower in wavelength. For the modes relevant to the vertical impedance $\left({ }_{\mathrm{C}} \mathrm{H}_{11}\right.$ and $\left.\mathrm{H}_{10}\right)$, the cutoff wavelength grows linearly (approximately for ${ }_{\mathrm{C}} \mathrm{H}_{11}$ ) with $a$; the proportionality coefficient is $\sim 3.33$ for elliptical vs 4 for rectangular. On the other hand, at large values of $a / b$, the cutoff wavelengths for the modes relevant to the horizontal and quadrupolar impedances become independent of $a / b$ and asymptotically approach $4 b$ (for $\mathrm{H}_{01}$ this value is exact at any aspect ratio).

These observations allow us to (approximately) limit the upper frequency extent of the inductive regime for the elliptical cross-section structure by

$$
\begin{gathered}
f_{\max } \cong 0.3 \frac{c}{a_{\min }} \quad \text { (vertical) } \\
f_{\max } \cong \frac{c}{4 b_{\min }} \text { (horizontal and quadrupolar). }
\end{gathered}
$$

In Eq. (2.13) for the sake of simplicity we are ignoring a weak aspect-ratio dependence of the cutoff frequency (see Fig. 9).

It is worth emphasizing that to our knowledge this result is new, and it is different from what has been suggested before in two important aspects. First of all, we find that the scaling with structure dimensions is different for the ver- 
tical impedance as opposed to the horizontal and quadrupolar. As a result, for large aspect-ratio structures the inductive range in the vertical is much narrower. Second, the upper frequency for the vertical impedance given by Eq. (2.12) is inversely proportional to the structure width and is independent of the taper length $L$. This is qualitatively different from the condition suggested in [6] [Eq. (6)] for the vertical-dipolar impedance of the rectangular structure of constant half-width $w$. In [6] it is stated that the inductive regime extends up to $f_{\max } \cong \pi c L / w^{2}$.

We should mention that since the arguments presented here are quite general we expect the conclusions relating the extent of the inductive impedance to the cutoff frequency of the corresponding mode to hold for any crosssection shape, not just elliptical. For example, for the rectangular case, we believe that the inductive regime is limited from above by the cutoff frequencies given by Eqs. (2.9), (2.10), and (2.11).

\section{E. Kick factor}

Let us now discuss the kick factor, which is a key quantity characterizing the transverse single bunch dynamics [24]. For a bunch passing through the structure, the kick factor $\kappa_{u}(u=x, y)$ relates the bunch-centroid deflection $\left\langle u^{\prime}\right\rangle$ to the bunch-centroid displacement $\langle u\rangle$ via

$$
\left\langle u^{\prime}\right\rangle=\frac{4 \pi Q}{Z_{0} c E / e} \kappa_{u}\langle u\rangle,
$$

where $Q$ and $E$ are the bunch charge and energy, respectively. In this definition, a positive kick factor corresponds to a defocusing force. The total kick factors are related to the dipolar and quadrupolar kick factors by $\kappa_{x}=\kappa_{D x}+$ $\kappa_{Q}$ and $\kappa_{y}=\kappa_{D y}-\kappa_{Q}$. The kick factors are related to the transverse impedance or the wake potential by

$$
\begin{aligned}
\kappa_{D x, D y, Q} & =-\frac{1}{\pi} \int_{0}^{\infty} d \omega|\tilde{f}(\omega)|^{2} \operatorname{Im}\left[Z_{D x, D y, Q}(\omega)\right] \\
& =\int_{-\infty}^{\infty} d z f(z) W_{D x, D y, Q}(z)
\end{aligned}
$$

where $f(z)$ is unity normalized longitudinal beam distribution, $\tilde{f}(\omega)$ is its Fourier transform, and $W_{D x, D y, Q}(z)$ is the wake potential due to this distribution. While in the total beam deflection [Eq. (2.14)] dipolar and quadrupolar kick factors simply add together, they play different roles in collective beam dynamics. The dipolar component may contribute to instability while the quadrupolar part may provide a stabilizing influence by adding some frequency spread $[9,12]$.

Performing numerical integration of the right-hand side (rhs) of Eq. (2.15), we have calculated the vertical-dipolar kick factor for a $1 \mathrm{~cm}$ rms long driving Gaussian bunch from the GDFIDL wake potentials shown earlier in Fig. 4. Then, by making use of basic Fourier transform properties, we obtained the kick factors for longer bunches (starting again from the same wake potentials due to $\sigma_{z}=1 \mathrm{~cm}$ bunch).

The resulting kick factors are plotted in Fig. 10(a). For small aspect ratios, the dominant scaling with the bunch length is $\kappa_{D y} \sim 1 / \sigma_{z}$. This is expected as these cases belong to the inductive regime where the kick factor is simply given by

$$
\kappa_{D y} \cong-\frac{c}{2 \sqrt{\pi} \sigma_{z}} \operatorname{Im}\left[Z_{D y}(0)\right]
$$

The cases of large aspect ratios also fall into this regime for longer bunches, but break out of it when the bunch length satisfies the condition
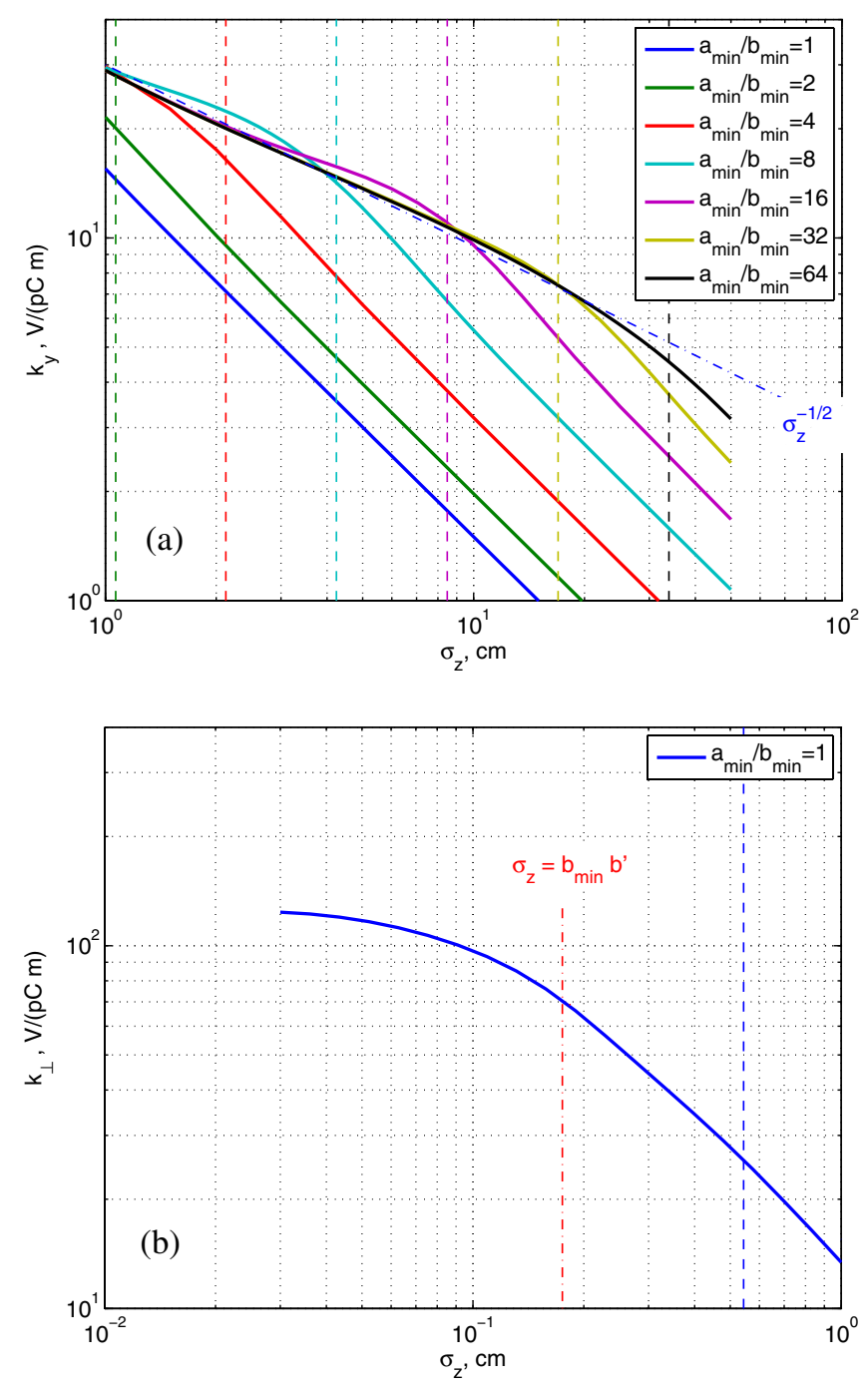

FIG. 10. (Color) (a) Vertical-dipolar kick factor vs bunch length derived from GDFIDL wake potentials due to a $1 \mathrm{~cm}$ rms bunch (solid line). Threshold values of bunch length from Eq. (2.17) (color dashed line). (b) Transverse kick factor vs bunch length derived from $\mathrm{ABCI}$ wake potentials due to a $0.3 \mathrm{~mm}$ rms bunch in the axially symmetric structure (solid line). Bunch length from Eq. (2.17) (blue dashed line). 


$$
k_{c}^{\mathrm{c}^{\mathrm{H}}}{ }_{11}\left(a_{\min }, b_{\min }\right) \sigma_{z} \approx 1
$$

where $k_{c}^{\mathrm{c}^{\mathrm{H}_{11}}}\left(a_{\min }, b_{\min }\right) \approx 2 / a_{\min }$ is the cutoff wave number for the $\mathrm{C}_{\mathrm{C}} \mathrm{H}_{11}$ mode at the minimum cross section. This condition is shown in vertical dashed lines of corresponding color in Fig. 10 for each aspect ratio. Note that the $\kappa_{D y}\left(\sigma_{z}\right)$ dependence "break point" locations (where it deviates from inductive behavior) are given by Eq. (2.17). This is consistent with Eq. (2.12) and Fig. 9. We emphasize that the shortest bunch length for which the kick factor manifests inductive behavior scales inversely proportional to the structure width, not the width squared as suggested in [6].

For shorter bunches the kick factor has a weaker dependence on the bunch length than in the inductive regime. In fact, for the curves in Fig. 10(a) that correspond to the aspect ratios of 4 and higher, the short bunch scaling is close to $\kappa_{D y} \sim \sigma_{z}^{-1 / 2}$ (this scaling is shown in the dotdashed line), which is expected in the so-called "intermediate regime" [6]. In this regime, the kick factor is expected to be independent of the structure width, which is consistent with Fig. 10(a). Probing quantitative agreement is somewhat more difficult since, to our knowledge, there is no analytical expression for the kick factor of an elliptical taper in the intermediate regime. Estimating the kick factor at $\sigma_{z}=1 \mathrm{~cm}$ by the intermediate regime expression derived in [6] ${ }^{4}$ for a constant width rectangular cross-section taper results in a factor of 1.4 higher kick factor than what is shown in Fig. 10(a).

Finally, we note that the shorter the bunch the less is the overall growth in the kick factor as the structure changes from round (where the kick factor is inductive) to flat (intermediate). For example, at $\sigma_{z}=1 \mathrm{~cm}$, the kick factor of a flat structure is about twice the value for the round structure.

Our discussion so far has been focused on the behavior of the vertical-dipolar kick factor for structures with large aspect ratio. A separate comment about the extent of the inductive behavior of this kick factor for small aspect-ratio structures is in order. The two lowest aspect-ratio curves in Fig. 10(a), $a_{\min } / b_{\min }=1$ and $a_{\min } / b_{\min }=2$, exhibit inductive behavior for the entire bunch length interval shown; therefore the bunch length in our GDFIDL calculations is too long to establish the extent of the inductive behavior in these cases. On the other hand, for axially symmetric structures the issue has been resolved through analytical considerations in $[1,3,6]$ and has been confirmed by EM calculations [26]. It was found that for round structures the intermediate regime does not apply and, as bunches get shorter, the kick factor transitions from the

\footnotetext{
${ }^{4}$ Equation (16) of [6] misses a factor of $\frac{1}{2}$ which is mentioned in [25]. Our estimate uses the corrected expression. We match the minimum rectangular and elliptical cross sections as well as the height variation along both structures.
}

inductive behavior directly into the "diffraction regime," where $\kappa_{D y}$ does not depend on $\sigma_{z}$. For the axially symmetric version of our geometry this is illustrated in Fig. 10(b), which is calculated similar to Fig. 10(a), except the initial wake potential is from an ABCI [27] calculation for a $\sigma_{z}=0.3 \mathrm{~mm}$ driving Gaussian bunch. We note that Eq. (2.17) no longer accurately describes the extent of the inductive behavior for the kick factor; the condition $\sigma_{z} \sim$ $b_{\min } b^{\prime}$ (related to the $k r_{\min } r^{\prime} \ll 1$ condition of [1]) works much better for an axially symmetric structure. For axially asymmetric structures with small aspect ratio, it is conceivable that the kick factor behavior transitions between that shown in Fig. 10(a) to that of a round structure in Fig. 10(b), i.e., as $a_{\min } / b_{\min }$ gets closer to unity, the intermediate range becomes narrower and narrower and disappears completely for a round structure.

Finally, by examining Figs. 5 and 6 we see that the dipolar-horizontal and quadrupolar impedances behave in a similar manner to the transverse impedance of a round structure shown in Fig. 7, exhibiting inductive behavior over a broad frequency range. Therefore, we expect that the horizontal-dipolar and quadrupolar kick factors of asymmetric structures will behave in a similar manner to the kick factor of a round structure shown in Fig. 10(b).

\section{ANALYTIC CALCULATION OF IMPEDANCE AT ZERO FREQUENCY}

In order to find an analytic approximation to the lowfrequency transverse impedance we extend the approach originally suggested in [2] (for axially symmetric structures) to elliptical geometry. The main idea in this approach is that, in the inductive regime, the transverse impedance is well-approximated by its zero-frequency value, which is purely imaginary. Therefore, to good approximation, the problem of finding the transverse impedance reduces to the solution of electrostatic and magnetostatic problems.

Let us first consider the calculation of the horizontaldipolar impedance. To determine the electric (magnetic) field, we consider two displaced filaments with line charge (current) $\pm q$, comprising an electric (magnetic) dipole. Assuming the filaments are displaced in the horizontal direction by $r_{b}= \pm \Delta / 2$, the horizontal-dipolar impedance is given by

$$
Z_{D x}(0)=-\frac{i Z_{0}}{4 \pi q \Delta} \int_{-\infty}^{\infty} d z\left(E_{x}-H_{y}\right) .
$$

Similarly, assuming the filaments are displaced vertically by $\pm \Delta / 2$, we get for the vertical-dipolar impedance

$$
Z_{D y}(0)=-\frac{i Z_{0}}{4 \pi q \Delta} \int_{-\infty}^{\infty} d z\left(E_{y}+H_{x}\right)
$$

We emphasize that, for the dipolar impedance equations above, electric and magnetic fields are evaluated on the $z$-axis. 
The quadrupolar impedance can be calculated in a similar manner. To determine the electric (magnetic) field, we consider a filament on the $z$-axis with line charge (current) $q$, and find the electric (magnetic) field at a position displaced transversely from the axis. For simplicity we can pick either purely horizontal or purely vertical displacement $\left(x_{0}\right.$ or $\left.y_{0}\right)$. Then the quadrupolar impedance is given by

$$
\begin{aligned}
& Z_{Q x}(0)=-\frac{i Z_{0}}{4 \pi q x_{0}} \int_{-\infty}^{\infty} d z\left(E_{x}-H_{y}\right)=Z_{Q}(0), \\
& Z_{Q y}(0)=-\frac{i Z_{0}}{4 \pi q y_{0}} \int_{-\infty}^{\infty} d z\left(E_{y}+H_{x}\right)=-Z_{Q}(0) .
\end{aligned}
$$

Matching boundary conditions for a translation invariant (uniform) elliptical beam pipe is done most easily in elliptic cylindrical coordinates $(\mu, \theta, z)$ shown in Fig. 11. The contour surfaces of constant $\mu$ are confocal-elliptical cylinders, while those of constant $\theta$ are confocal hyperbolic cylinders. The confocal cylinder $\mu=\rho$ forms the inner beam pipe boundary while the $z$-axis is directed along the center of the beam pipe.

The relationship between Cartesian and elliptic coordinates is given by

$$
\begin{aligned}
& x=A \cosh \mu \cos \theta \\
& y=A \sinh \mu \sin \theta \\
& z=z,
\end{aligned}
$$

where $2 A$ is the distance between the foci. The major and minor semiaxes of the elliptical cross section are $a=$ $A \cosh \rho$ and $b=A \sinh \rho$. The limiting case of a circular cross section of radius $r$ is given by $A=2 r e^{-\rho}(\rho \rightarrow \infty)$. The opposite limit $\rho \rightarrow 0$ approximates a flat pipe having half-width $A$ and half-height $A \rho$. A convenient measure of the deviation from circularity is provided by

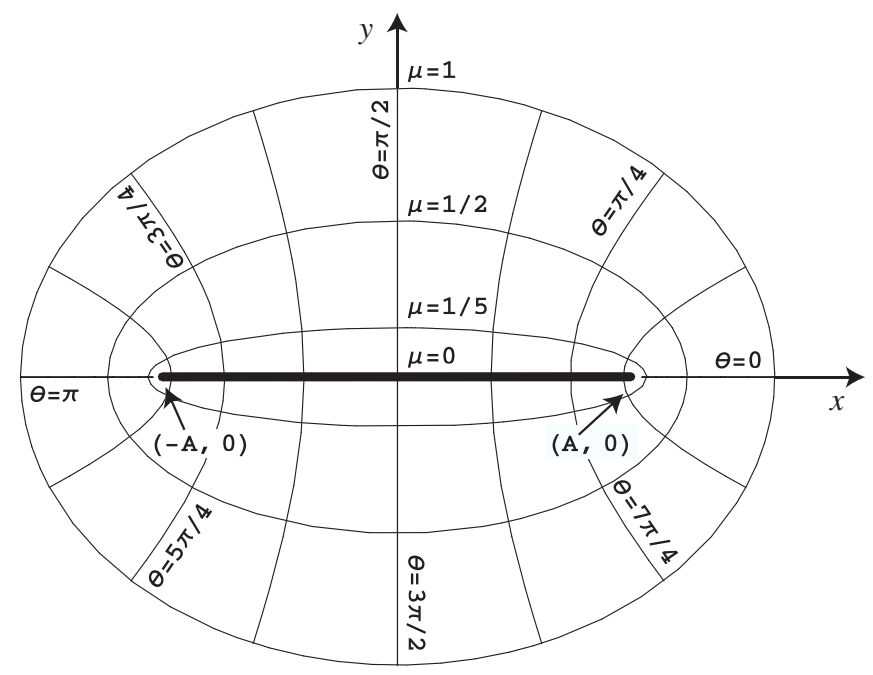

FIG. 11. Elliptic cylindrical coordinates.

$$
\alpha=\frac{a-b}{a+b}=e^{-2 \rho} .
$$

When the elliptical cross section varies with $z$, it is generally impossible to introduce an orthogonal coordinate system that matches the cross section at each value of $z$. However, in the case of confocal variation, we can still use the orthogonal elliptic coordinate system introduced above, which matches the beam pipe boundary greatly simplifying the boundary conditions. Specifically, we assume that $\rho$ is now a function of $z$, such that $\rho(z)=$ $\tanh ^{-1}[b(z) / a(z)]$. The parameter $A$ is given by

$$
A^{2}=a(z)^{2}-b(z)^{2} .
$$

The requirement for confocal variation is not very restrictive. It still allows arbitrary variation in one plane, for example, arbitrary beam pipe half-height $b(z)$. The variation in the other plane is then fixed as soon as $a(z)$ is specified at any single value of $z$.

\section{A. Vertical impedance}

Let us begin with the calculation of the vertical (dipolar) impedance $Z_{D y}$. We introduce the electrostatic potential $\phi$ so that the electric field $\vec{E}$ is expressed as $\vec{E}=-\nabla \phi$, and solve the Poisson equation with a vertical electric dipole source

$$
\nabla^{2} \phi=-4 \pi q \delta(x)\left[\delta\left(y-\frac{\Delta}{2}\right)-\delta\left(y+\frac{\Delta}{2}\right)\right] .
$$

We look for a solution that goes to zero at the elliptic metallic boundary and is continuous at the source-free points. Transforming to elliptic coordinates and assuming that $\Delta \ll A$, we derive the Poisson equation

$$
\begin{aligned}
& \frac{\partial^{2} \phi}{\partial \mu^{2}}+\frac{\partial^{2} \phi}{\partial \theta^{2}}+A^{2}\left(\sinh ^{2} \mu+\sin ^{2} \theta\right) \frac{\partial^{2} \phi}{\partial z^{2}} \\
& =-4 \pi q \delta\left(\mu-\frac{\Delta}{2 A}\right)\left[\delta\left(\theta-\frac{\pi}{2}\right)-\delta\left(\theta-\frac{3 \pi}{2}\right)\right]
\end{aligned}
$$

with the boundary condition

$$
\phi[\rho(z), \theta, z]=0 .
$$

Since the $z$-dependence only enters due to the boundary condition, the solution is a functional of $\rho(z)$, and we can write $\phi=\phi[\mu, \theta ; \rho(z)]$.

For a slowly varying beam pipe, $A \rho^{\prime}(z) \ll 1$, we can follow the approach suggested by Stupakov [2], and attempt to solve Eqs. (3.8) and (3.9) by using a perturbation expansion,

$$
\phi[\mu, \theta ; \rho(z)]=\phi^{0}[\mu, \theta ; \rho(z)]+\phi^{1}[\mu, \theta ; \rho(z)]+\cdots .
$$

Here $\phi^{0}=\phi^{0}\left[\mu, \theta ; \rho_{0}\right]$ is a solution of Eqs. (3.8) and (3.9) for a uniform beam pipe with $\rho(z)=\rho_{0}$, constant. It is 
straightforward to show that

$$
\begin{aligned}
\phi^{0}\left[\mu, \theta ; \rho_{0}\right]= & \frac{4 q \Delta}{A}\left[\frac{\sinh \mu \sin \theta}{\cosh 2 \mu+\cos 2 \theta}\right. \\
& \left.-\sum_{n=1}^{\infty} \sin \frac{n \pi}{2} e^{-n \rho_{0}} \frac{\sinh n \mu \sin n \theta}{\sinh n \rho_{0}}\right] .
\end{aligned}
$$

The first term is just the potential due to a vertical-dipole source in free space. The infinite sum consists of $\theta$-periodic solutions of the homogeneous Laplace equation chosen to match the boundary condition at $\mu=\rho_{0}$. Note that solutions of the Laplace equation of other types such as the ones proportional to $\cos n \theta$ do not appear due to the symmetry of the problem, while the terms $\cosh n \mu \sin n \theta$ cannot show up in $\phi^{0}$ due to the requirement that the potential is a continuous function of $\theta$ when $\mu=0$. For large $\rho_{0}$, one can drop all but the $n=1$ terms in the sum, and the above expression for $\phi^{0}$ correctly reduces to that of a circular pipe.

Proceeding to the next order in perturbation theory, we determine $\phi^{1}[\mu, \theta ; \rho(z)]$ by substituting Eq. (3.10) into Eq. (3.8), replacing the $\rho_{0}$ argument in $\phi^{0}$ with $\rho(z)$, yielding

$$
\frac{\partial^{2} \phi^{1}}{\partial \mu^{2}}+\frac{\partial^{2} \phi^{1}}{\partial \theta^{2}}=-A^{2}\left(\sinh ^{2} \mu+\sin ^{2} \theta\right) \frac{\partial^{2} \phi^{0}[\mu, \theta ; \rho(z)]}{\partial z^{2}},
$$

where we have ignored the second derivative $\partial^{2} \phi^{1} / \partial z^{2}$ on the right-hand side. After some algebra the solution to Eq. (3.12) which vanishes at $\mu=\rho(z)$ can be written as

$$
\begin{aligned}
\phi^{1}= & \frac{q \Delta A}{4} \sum_{n=1,3, \ldots}^{\infty} \sin \frac{\pi n}{2} \frac{D_{n}+D_{n+2}}{n+1} \\
& \times[\sinh (n+2) \mu \sinh n \rho-\sinh (n+2) \rho \sinh n \mu] \\
& \times\left[\frac{\sin n \theta}{\sinh n \rho}-\frac{\sin (n+2) \theta}{\sinh (n+2) \rho}\right]
\end{aligned}
$$

where we have defined

$$
D_{n}=\frac{\partial^{2}}{\partial z^{2}} \frac{e^{-n \rho(z)}}{\sinh n \rho(z)} .
$$

Analysis of the magnetostatic problem proceeds in a similar manner to that followed above for the electrostatic problem. We introduce a magnetostatic potential $\psi$ so that $\vec{H}=-\nabla \psi$, and solve the Poisson equation with a horizontal magnetic dipole source,

$$
\begin{aligned}
& \frac{\partial^{2} \psi}{\partial \mu^{2}}+\frac{\partial^{2} \psi}{\partial \theta^{2}}+A^{2}\left(\sinh ^{2} \mu+\sin ^{2} \theta\right) \frac{\partial^{2} \psi}{\partial z^{2}} \\
& \quad=-4 \pi q \delta(\mu)\left[\delta\left(\theta-\frac{\pi}{2}+\frac{\Delta}{2 A}\right)-\delta\left(\theta-\frac{\pi}{2}-\frac{\Delta}{2 A}\right)\right]
\end{aligned}
$$

with the boundary condition of zero normal magnetic field component on the wall

$$
\left[\frac{1}{A^{2}\left(\sinh ^{2} \mu+\sin ^{2} \theta\right)} \frac{\partial \psi}{\partial \mu}-\rho^{\prime}(z) \frac{\partial \psi}{\partial z}\right]_{\mu=\rho(z)}=0 .
$$

We proceed with the perturbation expansion as described previously. The zeroth-order solution $\psi^{0}\left[\mu, \theta ; \rho_{0}\right]$ is that corresponding to $\rho(z)=\rho_{0}$, constant. The boundary condition above reduces to $\left[\partial \psi^{0} / \partial \mu\right]_{\mu=\rho_{0}}=0$. It is straightforward to show that

$$
\begin{aligned}
\psi^{0}\left[\mu, \theta ; \rho_{0}\right]= & \frac{4 q \Delta}{A}\left[\frac{\cosh \mu \cos \theta}{\cosh 2 \mu+\cos 2 \theta}\right. \\
& \left.+\sum_{n=1}^{\infty} \sin \frac{n \pi}{2} e^{-n \rho_{0}} \frac{\cosh n \mu \cos n \theta}{\sinh n \rho_{0}}\right]
\end{aligned}
$$

To determine the first-order term $\psi^{1}[\mu, \theta ; \rho(z)]$, we must solve

$$
\frac{\partial^{2} \psi^{1}}{\partial \mu^{2}}+\frac{\partial^{2} \psi^{1}}{\partial \theta^{2}}=-A^{2}\left(\sinh ^{2} \mu+\sin ^{2} \theta\right) \frac{\partial^{2} \psi^{0}[\mu, \theta ; \rho(z)]}{\partial z^{2}},
$$

with the boundary condition

$$
\left[\frac{\partial \psi^{1}}{\partial \mu}-A^{2}\left(\sinh ^{2} \mu+\sin ^{2} \theta\right) \rho^{\prime}(z) \frac{\partial \psi^{0}[\mu, \theta ; \rho(z)]}{\partial z}\right]_{\mu=\rho(z)}=0 .
$$

This solution can be written as follows:

$$
\begin{aligned}
\psi^{1}= & -\frac{q \Delta A}{4} \sum_{n=1}^{\infty} \sin \frac{\pi n}{2}\left[\frac{D_{n}+D_{n+2}}{n+1}(\cosh (n+2) \mu \cos n \theta+\cosh n \mu \cos (n+2) \theta\right. \\
& \left.-\frac{(n+2) \sinh (n+2) \rho \cosh n \mu \cos n \theta}{n \sinh n \rho}-\frac{n \sinh n \rho \cosh (n+2) \mu \cos (n+2) \theta}{(n+2) \sinh (n+2) \rho}\right) \\
& \left.-4 \rho^{\prime}(z)\left(I_{n}+I_{n+2}\right)\left(\frac{\cosh (n+2) \rho \cosh n \mu \cos n \theta}{n \sinh n \rho}-\frac{\cosh n \rho \cosh (n+2) \mu \cos (n+2) \theta}{(n+2) \sinh (n+2) \rho}\right)\right]
\end{aligned}
$$


where we defined

$$
I_{n}=\frac{\partial}{\partial z} \frac{e^{-n \rho(z)}}{\sinh n \rho(z)}=-\frac{n \rho^{\prime}(z)}{\sinh ^{2} n \rho(z)} .
$$

Note that $D_{n}=\partial I_{n} / \partial z$.

In elliptic coordinates, the vertical impedance, Eq. (3.2), is determined by

$$
Z_{D y}(0)=\frac{i Z_{0}}{4 \pi A q \Delta} \int_{-\infty}^{\infty} d z\left[\frac{\partial \phi}{\partial \mu}-\frac{\partial \psi}{\partial \theta}\right]_{\mu=0, \theta=\pi / 2} .
$$

The impedance contributions from the zeroth-order potentials cancel out, correctly implying zero geometric impedance for a uniform pipe. Substituting the first-order potentials Eqs. (3.13) and (3.20) into Eq. (3.22) and integrating by parts assuming $\rho^{\prime}( \pm \infty)=0$, we find

$$
\begin{aligned}
Z_{D y}(0)= & \frac{i Z_{0}}{4 \pi} \sum_{n=1,3, \ldots}^{\infty} \int_{-\infty}^{\infty} d z \rho^{\prime}(z) \frac{I_{n}+I_{n+2}}{2(n+1)} \\
& \times\left(\frac{n \cosh (n+2) \rho}{\sinh n \rho}+\frac{(n+2) \cosh n \rho}{\sinh (n+2) \rho}\right. \\
& +\frac{n \sinh (n+2) \rho \cosh n \rho}{\sinh ^{2} n \rho} \\
& \left.+\frac{(n+2) \sinh n \rho \cosh (n+2) \rho}{\sinh ^{2}(n+2) \rho}\right) .
\end{aligned}
$$

Using Eq. (3.21), our final result for the vertical-dipolar impedance can be rewritten in the form

$$
\begin{aligned}
Z_{D y}(0)= & \frac{-i Z_{0}}{4 \pi} \sum_{n=1,3, \ldots} \int_{-\infty}^{\infty} d z \frac{\rho^{\prime}(z)^{2}}{2(n+1)}\left[\frac{n}{\sinh ^{2} n \rho(z)}\right. \\
& \left.+\frac{n+2}{\sinh ^{2}(n+2) \rho(z)}\right]^{2} \sinh 2(n+1) \rho(z) .
\end{aligned}
$$

We first look at the asymptotic limit of an almost circular pipe, $\rho(z) \gg 1$. Each subsequent term in the sum above is $O\left(e^{-2 \rho}\right)$ smaller than its predecessor, so one can easily write the impedance in powers of parameter $\alpha$ defined in Eq. (3.5). Limiting ourselves to the lowest-order correction we get

$$
\begin{gathered}
Z_{D y}(0) \cong-\frac{i Z_{0}}{2 \pi} \int_{-\infty}^{\infty} d z \rho^{\prime}(z)^{2}[1+4 \alpha(z)] \\
\text { for } \alpha(z) \ll 1
\end{gathered}
$$

Since for a circular pipe $r^{\prime}(z) / r(z)=\rho^{\prime}(z)$, the dominant term above is identical to the Yokoya expression Eq. (1.1) for an axially symmetric taper.

We now examine the opposite asymptotic limit of a flat pipe, $\rho(z) \ll 1$. In this case

$$
\begin{aligned}
Z_{D y}(0) \approx & \frac{-i Z_{0}}{4 \pi} \sum_{n=1,3, \ldots} \int_{-\infty}^{\infty} d z \frac{\rho^{\prime}(z)^{2}}{2(n+1)} \\
& \times\left[\frac{1}{n \rho^{2}}+\frac{1}{(n+2) \rho^{2}}\right]^{2} 2(n+1) \rho .
\end{aligned}
$$

Using the sum,

$$
\sum_{n=1,3, \ldots}\left[\frac{1}{n}+\frac{1}{n+2}\right]^{2}=\frac{\pi^{2}}{4},
$$

we find

$$
Z_{D y}(0) \approx \frac{-i Z_{0} \pi}{16} \int_{-\infty}^{\infty} d z \frac{\rho^{\prime}(z)^{2}}{\rho(z)^{3}} .
$$

For a flat elliptic pipe the width is almost constant and the half-height equals $A \rho$, so this expression differs from Stupakov's result for a flat rectangular pipe, Eq. (1.2), only by a factor of $\pi / 4$.

The intermediate region of medium eccentricities can be estimated from Eq. (3.24) directly by summing the nicely convergent series in MATHEMATICA [28].

\section{B. Horizontal impedance}

The horizontal impedance can be derived similarly to the vertical. Alternatively, one can make use of the transformation,

$$
\begin{aligned}
& \rho \rightarrow \rho+i \pi / 2, \\
& A \rightarrow-i A
\end{aligned}
$$

that interchanges the major and minor axes. This implies the relationship,

$$
Z_{x}(A, \rho)=Z_{y}(-i A, \rho+i \pi / 2) .
$$

Using the first-order perturbation result (3.24) for the vertical-dipolar impedance, we find that horizontal-dipolar impedance is determined by

$$
\begin{aligned}
Z_{D x}(0)= & \frac{-i Z_{0}}{4 \pi} \sum_{n=1,3, \ldots} \int_{-\infty}^{\infty} d z \frac{\rho^{\prime}(z)^{2}}{2(n+1)}\left[\frac{n}{\cosh ^{2} n \rho(z)}\right. \\
& \left.+\frac{n+2}{\cosh ^{2}(n+2) \rho(z)}\right]^{2} \sinh 2(n+1) \rho(z) .
\end{aligned}
$$

In the case of almost circular cross section, $\rho(z) \gg 1$, the impedance reduces to

$$
\begin{aligned}
Z_{D x}(0) \cong & -\frac{i Z_{0}}{2 \pi} \int_{-\infty}^{\infty} d z \rho^{\prime}(z)^{2}[1-4 \alpha(z)], \\
& \text { for } \alpha(z) \ll 1 .
\end{aligned}
$$

For the opposite case of a flat pipe, approximating the sum in Eq. (3.30) by an integral, we find

$$
Z_{D x}(0) \approx \frac{-i Z_{0}}{4 \pi} \int_{-\infty}^{\infty} d z \frac{\rho^{\prime}(z)^{2}}{\rho(z)^{2}} \quad \text { for } \rho(z) \ll 1,
$$


which is half of the Yokoya result Eq. (1.1) for an axially symmetric tapered transition with radius equal to the halfheight of the flat structure.

\section{Quadrupolar Impedance}

Let us now consider the quadrupolar impedance. The electric potential for a charged filament lying on the axis of a uniform elliptic chamber with constant $\rho(z)=\rho_{0}$ is given by

$$
\begin{aligned}
\phi^{0}= & 2 q\left(\rho_{0}-\mu\right)+q \sum_{n=1}^{\infty} \frac{4}{n}\left[e^{-n \mu}-e^{-n \rho_{0}} \frac{\cosh n \mu}{\cosh n \rho_{0}}\right] \\
& \times \cos \frac{n \pi}{2} \cos n \theta .
\end{aligned}
$$

For the perturbed wall, we must solve the equation (3.12), with the boundary condition $\phi^{1}[\rho(z), \theta ; \rho(z)]=0$. In this manner we determine the first-order electric potential

$$
\begin{aligned}
\phi^{1}= & -q\left(\rho^{\prime \prime}+\frac{B_{2}}{4}\right) \frac{A^{2}}{4}(\cosh 2 \mu-\cosh 2 \rho)\left(1-\frac{\cos 2 \theta}{\cosh 2 \rho}\right)+\frac{q A^{2}}{16} \sum_{n=1}^{\infty} \frac{B_{n}-B_{n+2}}{n+1}[\cosh (n+2) \mu \cosh n \rho \\
& -\cosh (n+2) \rho \cosh n \mu]\left[\frac{\cos n \theta}{\cosh n \rho}-\frac{\cos (n+2) \theta}{\cosh (n+2) \rho}\right]
\end{aligned}
$$

where

$$
B_{n}=\frac{4}{n} \frac{\partial^{2}}{\partial z^{2}} \frac{e^{-n \rho}}{\cosh n \rho} \cos \frac{n \pi}{2}
$$

The magnetic potential for a current filament along the axis of the uniform elliptic chamber with constant $\rho(z)=\rho_{0}$ is given by

$$
\psi^{0}=-2 q \theta-q \sum_{n=1}^{\infty} \frac{4}{n}\left[e^{-n \mu}+e^{-n \rho_{0}} \frac{\sinh n \mu}{\cosh n \rho_{0}}\right] \cos \frac{n \pi}{2} \sin n \theta
$$

For the perturbed wall, we must solve the equation (3.18) with the boundary condition (3.19). In this manner we determine the first-order magnetic potential

$$
\begin{aligned}
\psi^{1}= & \frac{q A^{2}}{16} \sum_{n=1}^{\infty}\left[\frac { B _ { n } - B _ { n + 2 } } { n + 1 } \left(\sinh (n+2) \mu \sin n \theta+\sinh n \mu \sin (n+2) \theta-\frac{(n+2) \cosh (n+2) \rho \sinh n \mu \sin n \theta}{n \cosh n \rho}\right.\right. \\
& \left.-\frac{n \cosh n \rho \sinh (n+2) \mu \sin (n+2) \theta}{(n+2) \cosh (n+2) \rho}\right)-4 \rho^{\prime}\left(C_{n}-C_{n+2}\right)\left(\frac{\sinh (n+2) \rho \sinh n \mu \sin n \theta}{n \cosh n \rho}\right. \\
& \left.\left.-\frac{\sinh n \rho \sinh (n+2) \mu \sin (n+2) \theta}{(n+2) \cosh (n+2) \rho}\right)\right]
\end{aligned}
$$

where

$$
C_{n}=\frac{4}{n} \frac{\partial}{\partial z} \frac{e^{-n \rho}}{\cosh n \rho} \cos \frac{n \pi}{2}
$$

Note that $B_{n}=\partial C_{n} / \partial z$.

The quadrupolar impedance could be found by

$$
\begin{aligned}
& Z_{Q x}(0)=-\frac{i Z_{0}}{4 \pi q x_{0} A} \int_{-\infty}^{\infty} d z\left[\frac{\partial \phi}{\partial \theta}+\frac{\partial \psi}{\partial \mu}\right]_{\mu=\cosh ^{-1}\left(x_{0} / A\right), \theta=0}=Z_{Q}(0), \\
& Z_{Q y}(0)=\frac{i Z_{0}}{4 \pi q y_{0} A} \int_{-\infty}^{\infty} d z\left[\frac{\partial \phi}{\partial \mu}-\frac{\partial \psi}{\partial \theta}\right]_{\mu=\sinh ^{-1}\left(y_{0} / A\right), \theta=\pi / 2}=-Z_{Q}(0),
\end{aligned}
$$

in the limit that $x_{0}$ or $y_{0}$ approach zero. Using the electric and magnetic potentials specified in Eqs. (3.34) and (3.37), we find within first-order perturbation theory,

$$
Z_{Q}(0)=\frac{i Z_{0}}{4 \pi} \sum_{n=0,2,4, \ldots} \int_{-\infty}^{\infty} d z \frac{\rho^{\prime}(z)^{2}}{2(n+1)}\left[\frac{1}{\cosh ^{2} n \rho}+\frac{1}{\cosh ^{2}(n+2) \rho}\right]\left[\frac{n^{2}}{\cosh ^{2} n \rho}+\frac{(n+2)^{2}}{\cosh ^{2}(n+2) \rho}\right] \sinh 2(n+1) \rho .
$$


In the limit of almost circular cross section,

$$
Z_{Q}(0) \cong \frac{i Z_{0}}{2 \pi} \frac{14}{3} \int_{-\infty}^{\infty} d z \rho^{\prime}(z)^{2} \alpha(z)
$$

where $\alpha(z) \ll 1$.

In the opposite limit of almost flat geometry,

$$
Z_{Q}(0) \cong \frac{i Z_{0}}{4 \pi} \int_{-\infty}^{\infty} d z \frac{\rho^{\prime}(z)^{2}}{\rho(z)^{2}}
$$

where $\rho(z) \ll 1$. Note that in this limit,

$$
Z_{D x}(0)+Z_{Q}(0) \cong 0 \text {. }
$$

The physical reason for this is clear. In a flat chamber, if the drive particle and the test particle are moved in the horizontal direction by the same amount, there can be no change in the horizontal force. If we take $x_{d}=x_{t}$ in Eq. (2.5), we see that $Z_{x}=0$ implies Eq. (3.43).

\section{HIGHER-ORDER PERTURBATION THEORY}

Let us now consider the conditions under which the firstorder perturbation theory will be a good approximation. Qualitatively this is easy to understand by examining the magnitude of the dropped terms in the magnetic boundary condition, Eq. (3.19), when going to the flat limit $\rho(z) \ll$ 1. Specifically, when solving for the first-order magnetic potential, we account for the term proportional to $\left[\frac{\partial \psi^{0}[\mu, \theta ; \rho(z)]}{\partial z}\right]_{\mu=\rho(z)}$ but neglect the one proportional to $\left[\frac{\partial \psi^{1}[\mu, \theta ; \rho(z)]}{\partial z}\right]_{\mu=\rho(z)}$. In the flat limit, the major semiaxis is almost constant, $a(z) \cong A$, and the minor semiaxis $b(z) \cong$ $A \rho(z)$. Now suppose the dependence of the minor semiaxis on the axial coordinate has the form,

$$
b(z)=b_{\mathrm{av}} F(z / L),
$$

where $b_{\mathrm{av}}$ is the average value of the minor semiaxis, and the smooth function $F(\xi)$ varies mainly in the interval $|\xi|<\sim 1$. Simple estimates show that, for the horizontal and quadrupolar impedances, the neglected terms will be small compared to those included if $b_{\mathrm{av}}^{2} / L^{2} \ll 1$. In contrast, for the vertical-dipolar impedance, the neglected terms will be small compared to those included in the first-order calculation only if $a^{2} / L^{2} \ll 1$. This implies that, for the horizontal and quadrupolar impedances, the first-order approximation will be accurate for all aspect ratios as long as the taper length is large compared to the minor semiaxis. On the other hand, for the vertical impedance, the requirement for validity of the first-order calculation is that the taper length be large compared to the major semiaxis, which is much more restrictive for flat structures.

A more rigorous approach to identify the small parameter (or parameters) that determines the validity of the firstorder approximation is to extend the perturbation theory to higher orders and compare the resulting higher-order impedance terms to each other. In the case of a circularly symmetric structure we have done this [4] and have shown that carrying out a perturbation expansion to order $n$ yields an asymptotic series in $\left(r_{\mathrm{av}} / L\right)^{2 n}$, i.e., we identified $\left(r_{\mathrm{av}} / L\right)^{2}$ as the small parameter.

Unfortunately, fully extending this approach to elliptical geometry is difficult. Specifically, to find the $(n+1)$ th order potentials from the $n$th order ones, we need to solve

$$
\begin{aligned}
\frac{\partial^{2} \phi^{n+1}}{\partial \mu^{2}}+\frac{\partial^{2} \phi^{n+1}}{\partial \theta^{2}}= & -A^{2}\left(\sinh ^{2} \mu+\sin ^{2} \theta\right) \\
& \times \frac{\partial^{2} \phi^{n}[\mu, \theta ; \rho(z)]}{\partial z^{2}} \\
\frac{\partial^{2} \psi^{n+1}}{\partial \mu^{2}}+\frac{\partial^{2} \psi^{n+1}}{\partial \theta^{2}}= & -A^{2}\left(\sinh ^{2} \mu+\sin ^{2} \theta\right) \\
& \times \frac{\partial^{2} \psi^{n}[\mu, \theta ; \rho(z)]}{\partial z^{2}},
\end{aligned}
$$

subject to the boundary conditions

$$
\begin{gathered}
\left.\phi^{n+1}[\mu, \theta ; \rho(z)]\right|_{\mu=\rho(z)}=0, \\
{\left[\frac{\partial \psi^{n+1}}{\partial \mu}-A^{2}\left(\sinh ^{2} \mu+\sin ^{2} \theta\right) \rho^{\prime}(z) \frac{\partial \psi^{n}[\mu, \theta ; \rho(z)]}{\partial z}\right]_{\mu=\rho(z)}} \\
=0 .
\end{gathered}
$$

These are linear partial differential equations (unlike ordinary differential equations for the circular case) and when the $n$th order potentials are given by infinite series [see, e.g., Eqs. (3.13) and (3.20) for $n=1$, vertical] MATHEMATICA cannot solve directly for the next-order potential.

On the other hand, since the equations are linear, the transformation of each term in the series for the potentials (as the perturbation theory order increases by one) is fairly straightforward, i.e., terms proportional to $\cos (n \theta)$ transform to $\cos [(n \pm 2) \theta]$ due to the rhs, additional terms proportional to $\cos (n \theta)$ appear due to boundary conditions. Putting these transformation rules explicitly into MATHEMATICA and having it to keep track of all the coefficients, we were able to solve for the second-order potentials and find the second-order impedances.

The resulting second-order potentials have been checked to obey the Poisson equations and the boundary conditions given above. Second-order dipolar impedances (too bulky to be presented here ${ }^{5}$ ) were also checked to reduce to the result of [4] in the limit of axially symmetric pipe. In this limit, the second-order quadrupolar impedances vanish.

\footnotetext{
${ }^{5}$ Note, that for nonsmooth structure boundaries such as Fig. 1, having explicit expressions for a finite number of higher-order impedances is not useful to improve the accuracy of the firstorder result. This is due to the fact that the derivatives of the boundary of orders higher than one are not integrable [4].
} 
In the limit of an almost flat structure, $\rho(z) \ll 1$, the impedances determined to second order are given by

$$
\begin{aligned}
& Z_{D x}(0)= \frac{-i Z_{0}}{4 \pi} \int_{-\infty}^{\infty} d z\left[\frac{\rho^{\prime}(z)^{2}}{\rho(z)^{2}}-A^{2}\left(\frac{5}{9} \frac{\rho^{\prime}(z)^{4}}{\rho(z)^{2}}\right.\right. \\
&\left.\left.+0.186 \rho^{\prime \prime}(z)^{2}\right)\right] \\
& Z_{Q}(0)=-Z_{D x}(0), \\
& Z_{D y}(0)=-\frac{i Z_{0} \pi}{16} \int_{-\infty}^{\infty} d z\left[\frac{\rho^{\prime}(z)^{2}}{\rho(z)^{3}}-A^{2} \frac{2 \pi^{2}-9}{144}\right. \\
&\left.\times\left(-5 \frac{\rho^{\prime}(z)^{4}}{\rho(z)^{5}}+3 \frac{\rho^{\prime \prime}(z)^{2}}{\rho(z)^{3}}\right)\right] .
\end{aligned}
$$

Let us assume the dependence of the minor semiaxis on the axial coordinate has the form given in Eq. (4.1). In this case, we see that the magnitudes of the second-order $\left(Z^{2}\right)$ contributions to the zero-frequency impedances relative to first-order $\left(Z^{1}\right)$ contributions are given by

$$
\begin{aligned}
& \lim _{b_{\mathrm{av}} / a \rightarrow 0} Z_{D x}^{2} / Z_{D x}^{1} \propto b_{\mathrm{av}}^{2} / L^{2}, \\
& \lim _{b_{\mathrm{av}} / a \rightarrow 0} Z_{Q}^{2} / Z_{Q}^{1} \propto b_{\mathrm{av}}^{2} / L^{2}, \\
& \lim _{b_{\mathrm{av}} / a \rightarrow 0} Z_{D y}^{2} / Z_{D y}^{1} \propto a^{2} / L^{2},
\end{aligned}
$$

in agreement with the simpler argument given earlier.

In summary, for the limiting case $(\rho \rightarrow 0)$ of a smooth flat chamber, we found that the horizontal and quadrupolar impedance second-order corrections can be neglected for taper length $L$, if the average chamber minor semiaxis satisfies $b_{\mathrm{av}}^{2} / L^{2} \ll 1$. In contrast, to assure that secondorder terms for the vertical-dipolar impedance are negligible, it is additionally required that the chamber major semiaxis satisfies $a_{\mathrm{av}}^{2} / L^{2} \ll 1$. Therefore, the perturbation theory breaks down when the taper width is comparable to or larger than its length.

We also comment that our conclusions, Eqs. (4.9), (4.10), and (4.11), are strictly valid for smooth structures only. Boundaries that include corners such as shown in Fig. 1 do not have integrable derivatives of orders higher than one, preventing a straightforward evaluation of the higher-order terms. Our previous work with the axially symmetric case [4] suggests that, for such structures, corrections due to higher-order impedances sum up to a contribution proportional to the 1 st power of the small parameter (vs the 2 nd power for a smooth case).

\section{COMPARISON OF FIRST-ORDER PERTURBATION THEORY TO GDFIDL RESULTS}

As we discussed in Sec. II, the beam dynamics in the inductive regime is approximately characterized by the impedance at zero frequency. Here, we compare the approximation derived using first-order perturbation theory for the zero-frequency impedance (Sec. III) with the results of GDFIDL calculations. For brevity we drop the (zero) frequency argument of the impedance. Results are presented in Figs. 12-14.

Impedances are normalized by the GDFIDL values of dipolar impedance, $Z_{\text {round }}$, for a pipe with circular cross section having the same vertical profile as the elliptic chamber under consideration. We plot this normalized impedance vs the ellipse aspect ratio at the smallest cross section, $a_{\min } / b_{\min }$. We present two plots for the vertical impedance (Fig. 12): (a) linear scale and small range
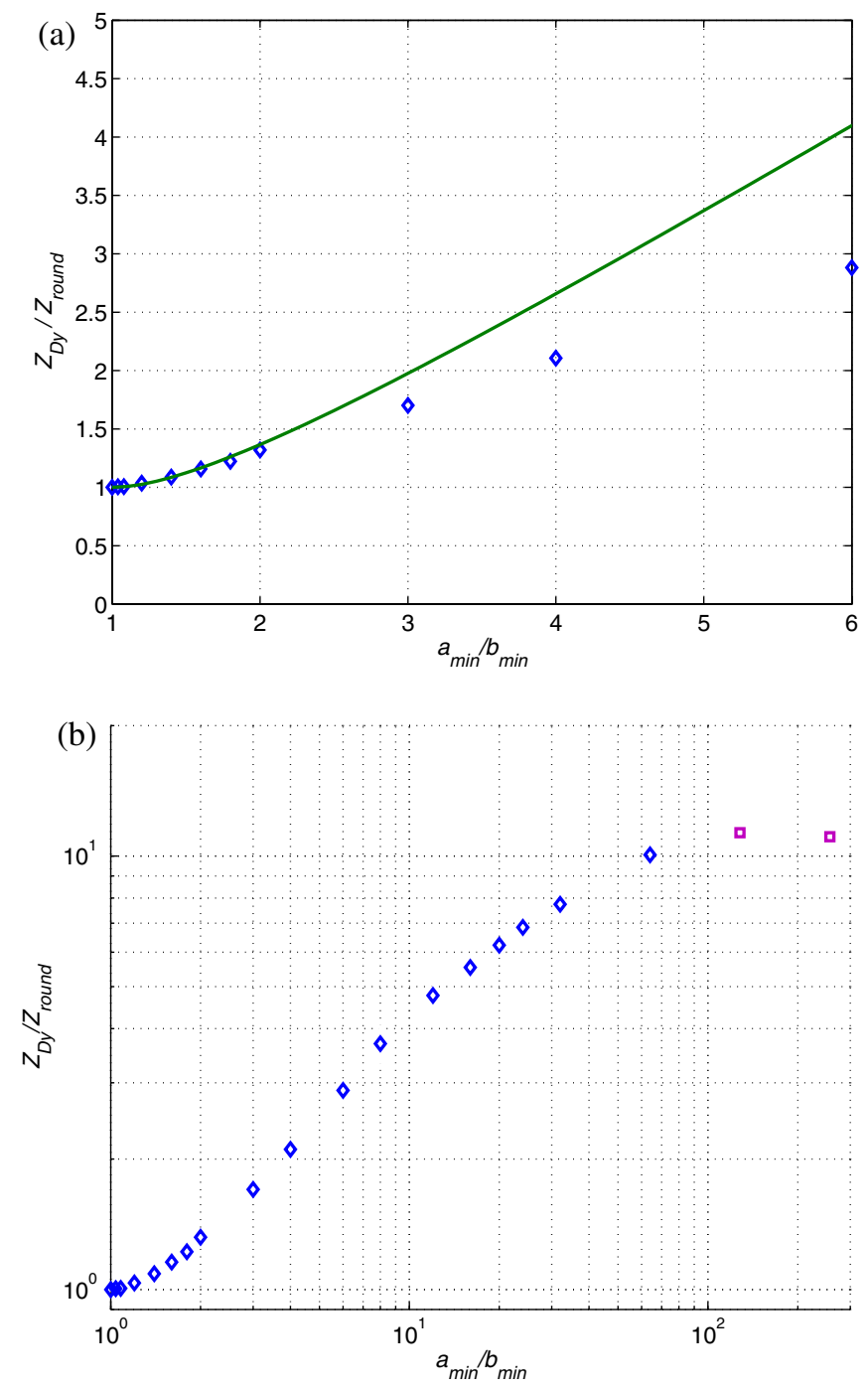

FIG. 12. (Color) Zero-frequency vertical impedance from GDFIDL (symbols) and from the first-order perturbation theory Eq. (3.24) (solid line). 


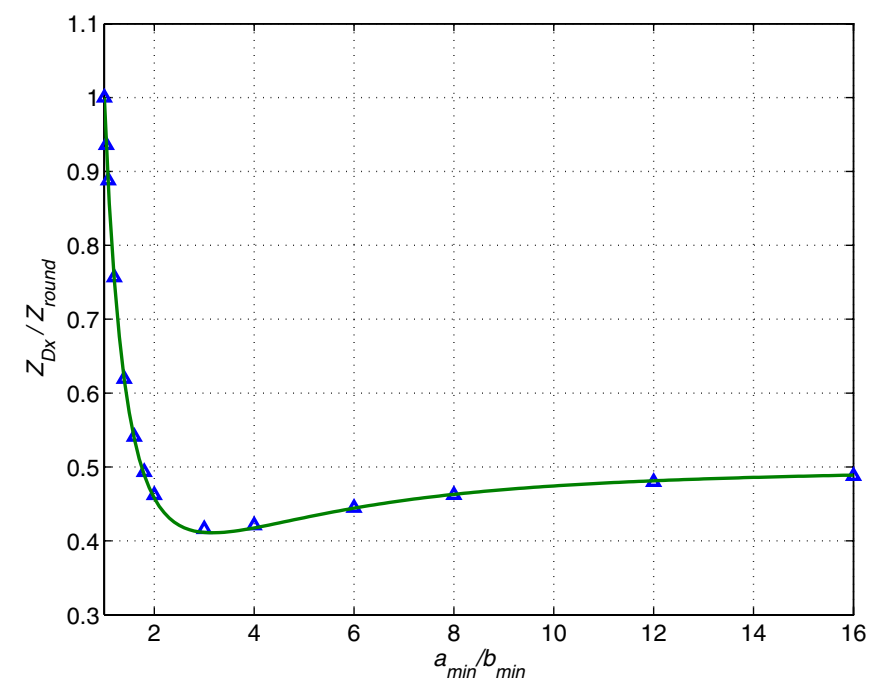

FIG. 13. (Color) Zero-frequency horizontal impedance from GDFIDL (symbols) and from first-order perturbation theory Eq. (3.30) (solid line).

$a_{\min } / b_{\min }<6$ to provide detailed comparison with theory, and (b) logarithmic scale to show the behavior at large aspect ratios. Good agreement with GDFIDL is observed up to aspect ratio of $\sim 3$, where $a_{\mathrm{av}}^{2} / L^{2} \approx 0.17$. Both GDFIDL and perturbation theory show a regime of linear increase of the impedance with aspect ratio; however, the perturbation approximation appears to predict a slope which is about $25 \%$ too large. Also, it is seen from Fig. 12(b) that the vertical impedance clearly exhibits saturation at large aspect ratios not predicted by the first-order theory.

On the other hand, the horizontal and quadrupolar impedances (Figs. 13 and 14) exhibit excellent agreement with first-order perturbation theory for all aspect ratios. For the gradually tapered structure being analyzed $\left(b_{\mathrm{av}}^{2} / L^{2} \approx\right.$ 0.076), this excellent agreement for the horizontal and

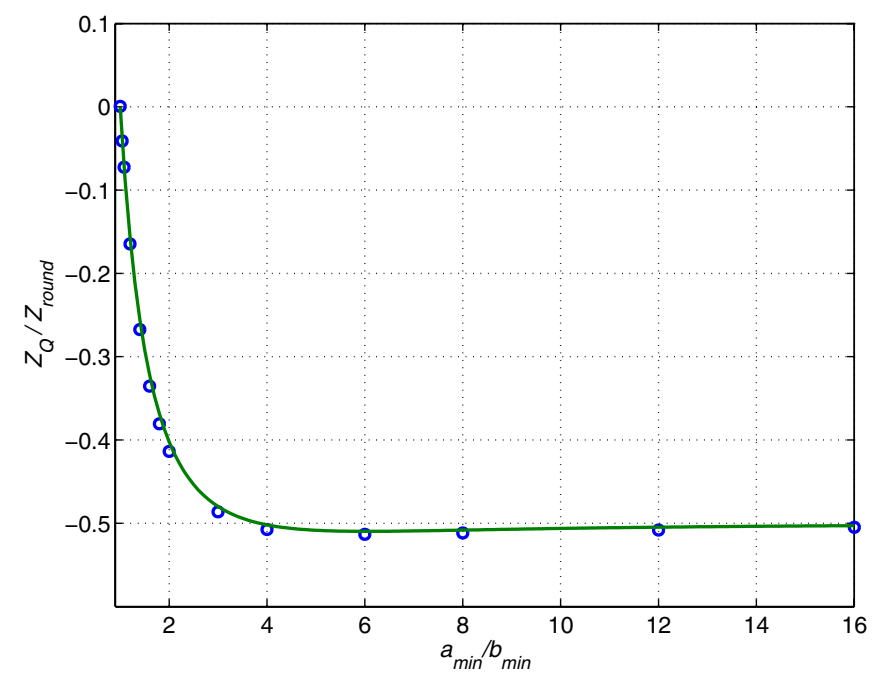

FIG. 14. (Color) Zero-frequency quadrupolar impedance from GDFIDL (symbols) and from first-order perturbation theory Eq. (3.40) (solid line). quadrupolar impedances and limited agreement for the vertical impedance is consistent with the discussion presented in Sec. IV. Interestingly, both $Z_{D x}$ and $Z_{Q}$ reach their "flat limit" at rather low aspect ratio $\sim 2-3$. Similar behavior was found for the resistive wall impedance in uniform pipes with elliptical and rectangular cross sections $[18,29]$.

\section{SUMMARY}

We studied the transverse geometric impedance of gradually tapered structures with elliptic cross section. First-order perturbation results at zero-frequency were obtained for the vertical-dipolar, horizontal-dipolar, and quadrupolar impedances, given in Eqs. (3.24), (3.30), and (3.40), respectively. Consideration of the perturbation expansion to second order suggested that the first-order approximation for horizontal and quadrupolar impedances is accurate for arbitrary structure widths, as long as the height variation is gradual, i.e. $b_{\mathrm{av}}^{2} \ll L^{2}$. On the other hand, the condition that the vertical impedance is well approximated by the first-order result requires that a more stringent condition, $a^{2} \ll L^{2}$, be satisfied. When the vertical variation is gradual, but $a \sim L$, the higher-order terms become significant and reduce the vertical impedance.

The first-order results reduce to simple asymptotic expressions in the limit of "almost round" [Eqs. (3.25), (3.31), and (3.41)] and "almost flat" structures [Eqs. (3.27), (3.32), and (3.42)]. Specifically, in the flat limit, the horizontal and quadrupolar impedances are given, respectively, by one-half and minus one-half of the Yokoya impedance Eq. (1.1) of a round structure having the same vertical profile. The vertical impedance in the flat limit, given by Eq. (3.27), exhibits a linear dependence on width, $Z_{D y} \propto a$, which is similar to the result [3] for rectangular cross-sectioned tapers. The proportionality coefficient we have determined for the wide elliptical taper is a factor $\pi / 4$ smaller than the one found in [3] for the rectangular case.

For the simple case of a single taper with linear vertical tapering,

$$
b(z)=\left\{\begin{array}{cc}
b_{\min }=b_{\mathrm{av}}(1-\varepsilon) & (z \leq-L) \\
b_{\mathrm{av}}\left(1+\frac{\varepsilon z}{L}\right) & (-L<z<L) \\
b_{\max }=b_{\mathrm{av}}(1+\varepsilon) & (L \leq z)
\end{array}\right\},
$$

with $0<\varepsilon<1$, and large aspect ratio, $a \cong a_{\mathrm{av}} \cong A \gg$ $b_{\mathrm{av}}$, the impedances are given by

$$
\begin{gathered}
Z_{D x}(0)=-Z_{Q}(0) \cong-\frac{i Z_{0}}{2 \pi L} \frac{\varepsilon^{2}}{1-\varepsilon^{2}}, \\
Z_{D y}(0)=-\frac{i \pi Z_{0}}{8 L} \frac{\varepsilon^{2}}{\left(1-\varepsilon^{2}\right)^{2}} \frac{a_{\mathrm{av}}}{b_{\mathrm{av}}}
\end{gathered}
$$

Equations (6.2) and (6.3) should be multiplied by 2 for a dual taper structure such as that of Fig. 1. 
Our first-order formulas, Eqs. (3.24), (3.30), and (3.40), approach their asymptotic "flat structure behavior" at rather modest aspect ratios (see, e.g., analytical curves in Figs. 12-14). This and other features of impedance behavior vs the structure aspect ratio are very similar to the qualitative features found for the resistive wall impedance $[18,29]$.

Our first-order perturbation results discussed above were compared to GDFIDL calculations and for the horizontaldipolar and quadrupolar components the agreement was found to be excellent. On the other hand, for the vertical impedance good agreement was found only for modest aspect ratios. Here, we were not able to fully verify the flat limit, Eq. (3.27), because limited computer resources did not allow us to use sufficiently large taper length, to satisfy $h_{\mathrm{av}}^{2} \ll a^{2} \ll L^{2}$. Nevertheless, the calculations we have performed with the restricted geometry showed the predicted proportionality, $Z_{D y} \propto a$, but with a roughly $25 \%$ lower proportionality coefficient than predicted by Eq. (3.27). The linear behavior exhibited by the GDFIDL results extended roughly to $a \sim L$; at larger widths, GDFIDL showed saturation of the increase of the vertical impedance with major semiaxis $a$, qualitatively consistent with the effect due to higher-order terms in the perturbation theory.

By means of GDFIDL calculations, we have established that the inductive regime extends from $\mathrm{DC}$ to $f_{\max }$, where $f_{\max }$ is the lowest cutoff frequency of the waveguide formed by the smallest cross section for the H-mode that has appropriate symmetry and direction of the EM field; at frequencies exceeding $f_{\max }$ a significant real part of impedance appears. For elliptical structure, the modes for the vertical, horizontal, and quadrupolar cases are ${ }_{\mathrm{C}} \mathrm{H}_{11},{ }_{\mathrm{S}} \mathrm{H}_{11}$, and ${ }_{5} \mathrm{H}_{21}$, respectively. Consequently, when $a_{\min } \gg b_{\min }$, the cutoff frequencies scale approximately as $c / 4 b_{\min }$ (horizontal and quadrupolar) and $0.3 c / a_{\min }$ (vertical). Therefore, for flat structures the frequency range of the inductive regime is much smaller for the vertical dipolar than for the horizontal dipolar and quadrupolar impedance components. In addition to this significant difference in the extent of inductive ranges for the three impedance components, we found that these ranges do not depend on the taper length (unlike the criteria suggested previously), but depend only on the dimensions of the minimal cross section.

We also studied the dependence of the vertical dipolar kick factor on bunch length, and found that, unless the structure is close to round, this dependence transitions from inductive $\left(\kappa_{D y} \sim \sigma_{z}^{-1}\right)$ to intermediate $\left(\kappa_{D y} \sim\right.$ $\left.\sigma_{z}^{-(1 / 2)}\right)$ behavior at bunch length values corresponding to the ${ }_{\mathrm{C}} \mathrm{H}_{11}$ mode cutoff frequency mentioned above, see Eq. (2.17). When the structure is axially symmetric (or close to it), the kick factor continues its inductive behavior down to the bunch length given by $\sigma_{z} \cong b^{\prime} b_{\min }$, after which it approaches the diffractive $\kappa_{D y}\left(\sigma_{z}\right)=$ const limit.
A related observation from GDFIDL was a growth saturation of the vertical short-range wake (as a function of the structure width) when the bunch was short enough to probe higher frequencies beyond the inductive regime, so that the kick factor was transitioning to the intermediate behavior. For $\sigma_{z}=1 \mathrm{~cm}$, the peak value of the short-range vertical wake potential (and the vertical kick factor) plateaued at about twice the round structure value (see Figs. 4 and 10). The implication of the short-range wake plateauing at rather modest values is that single bunch effects do not become too strong as the aspect ratio increases. On the other hand, the long range wake which appears for wide structures may contribute to coupled bunch effects and needs to be estimated carefully.

Finally, our results obtained for confocal-elliptical structures, given in Eqs. (3.27), (3.32), and (3.42), can provide reasonable estimates for gradual tapers with crosssectional shapes other than elliptical, as long as they have a large enough horizontal-to-vertical aspect ratio at the minimum cross section. To proceed, one considers a corresponding confocal-elliptical structure with $b(z)$ matched everywhere to the vertical aperture of the taper and $a(z)$ matched to the horizontal aperture at the axial position $z$ where the horizontal aperture is minimum. We have performed several GDFIDL runs for varied gradual taper geometries and found good agreement using this recipe.

\section{ACKNOWLEDGMENTS}

We thank A. Blednykh for stimulating discussions about his work on trapped modes [22] and on the use of GDFIDL. Support on the utilization of GDFIDL provided by W. Bruns [7] has been of great importance. We wish to thank K. Bane, P. J. Chou, and G. V. Stupakov for clarifying discussions, and I. Zagorodnov for providing important confirmation of a subset of our numerical results by running his code ECHO [31]. Our work was supported by Department of Energy Contract No. DE-AC0298CH10886.

\section{APPENDIX: DETAILS OF GDFIDL CALCULATIONS}

As it was already mentioned in Sec. II, for our GDFIDL calculations we have chosen a basic piecewise linear tapered structure shown in Fig. 1. The main reason for this geometry was that we have already studied its axially symmetric version in detail [4], both analytically and numerically utilizing ABCI [27]. Original arguments for this particular geometry were as follows. Since we are studying the impedance of a taper (rather than possible interference between two tapers), we have chosen the length of the middle section to be long enough to assure that its lengthening does not affect the results. In other words, this geometry is suitable for studying the regime of two separate steps. Also, we have chosen long enough 
outer pipes to not affect our results. Finally, we picked a convex geometry (Fig. 1), that resulted in lesser amount of numerical noise, and therefore permitted us to use a more coarse mesh. Note that both ABCI and GDFIDL allow for concave structures by utilizing some version of Napoly integration [30]; however, we found the results for the zero-frequency impedance calculated in such manner to be less accurate. Nevertheless, we did check for several aspect ratios that in the limit of vanishing mesh size the results for the low-frequency broadband impedance for convex and concave configurations are essentially the same, which is consistent with the physical intuition.

GDFIDL allows for arbitrary transverse position of the drive charge within a structure as well as arbitrary transverse offset for the path to integrate the resulting fields for the wake potential. We separately calculate the horizontal and vertical-dipolar wake potentials by displacing a $\sigma_{z}=$ $1 \mathrm{~cm} \mathrm{rms} \mathrm{long} \mathrm{bunch} \mathrm{by} \Delta_{x}$ or $\Delta_{y}$ in the appropriate plane and integrating the on-axis fields. To calculate the quadrupolar wake potential, we place the drive charge on-axis and integrate the off-axis fields with either a purely vertical or purely horizontal displacement $\Delta_{Q}$. In both cases the results for $Z_{Q}$ came out essentially the same.

We take advantage of the symmetry planes and perform the calculations for a quarter structure, $x>0, y>0$, enforcing the electric boundary condition in the $y=0(x=$ 0 ) plane and the magnetic boundary condition in the $x=0$ $(y=0)$ plane when calculating vertical (horizontal) dipolar wake potentials. Note that, due to the electric boundary condition (and small offsets $\Delta_{x}=\Delta_{y}=1.2 \mathrm{~mm}$ we typically used), integrating the fields on-axis or along the (offset) path of the drive charge resulted in virtually the same wake potentials. Both boundaries were set to magnetic for quadrupolar wake calculations.

The wake potentials were calculated up to $s_{\max }=2 \mathrm{~m}$ distance behind the drive bunch, with the exception of $W_{y}$ for large aspect-ratio structures, where $s_{\max }$ had to be extended to $5 \mathrm{~m}$ so that the ringing sufficiently damps down to not affect the zero-frequency impedance by more than a few percent. To get the zero-frequency impedance presented in Figs. 12-14, we numerically integrated the wake potential up to distance $s_{\max }$ behind the drive bunch and normalized the result to the $\Delta_{x, y}$ in the dipolar case or $\Delta_{Q}$ for quadrupolar impedances,

$$
Z_{x, y, Q}(0)=\frac{1}{c q \Delta_{x, y, Q}} \int_{-6 \sigma_{z}}^{s_{\max }} d z W_{x, y, Q}^{\mathrm{GDFIDL}}(z)
$$

where $W^{\text {GDFIDL }}$ [Volts] is the corresponding wake potential due to the drive charge $q$.

Given our computer resources, the smallest mesh size we could use and yet go for rather high aspect ratio, $a_{\min } / b_{\min }=64$, was $400 \mu \mathrm{m}$. All results presented in this paper are calculated with this mesh size [with the exception of two highest aspect ratio points, $a_{\min } / b_{\min }=$ 128 and $a_{\min } / b_{\min }=256$, in Fig. 12(b) where we used 500 and $667 \mu \mathrm{m}$ mesh sizes, respectively]. To ensure this mesh size was adequate we performed checks described below. First, we compared GDFIDL results for axially symmetric structure with $\mathrm{ABCI}$ in the range of $250-500 \mu \mathrm{m}$ mesh sizes. The agreement was found to be perfect; the dependence on the mesh size was linear, its zero mesh size extrapolation (which we believe is close to the true value of the impedance) was $1.53 \mathrm{k} \Omega / \mathrm{m}$ [4]. With our nominal mesh size of $400 \mu \mathrm{m}$ GDFIDL (and ABCI) value is $\sim 15 \%$ higher. Performing similar GDFIDL calculations for $a_{\min } / b_{\min }=4$ structure, we established that the mesh size dependence of $Z(0)$ is again linear, and that the relative change in $Z(0)$ with mesh size changing from 250 to $400 \mu \mathrm{m}$ was essentially the same as for the round structure. This is why we believe that our main results presented in Figs. 12-14 are accurate to a few percent, as we normalize the impedance at every aspect ratio to that of the axially symmetric structure (also calculated by GDFIDL at the same nominal mesh size) so the systematic errors due to finite mesh size largely cancel out.

We also performed a number of other consistency checks, such as linearity of $W_{x, y, Q}^{\mathrm{GDFIDL}}$ with the driving or trailing charge displacements, independence of $Z(0)$ from the drive bunch length, etc., and found the errors due to them to be negligible. Finally, Zagorodnov has provided an important confirmation of a subset of our numerical results by running his code ECHO [31].

[1] K. Yokoya, CERN SL/90-88 (AP).

[2] G. V. Stupakov, SLAC-PUB-7086.

[3] G. V. Stupakov, SLAC-PUB-7167.

[4] B. Podobedov and S. Krinsky, Phys. Rev. ST Accel. Beams 9, 054401 (2006).

[5] G. V. Stupakov (private communication).

[6] G.V. Stupakov, Proceedings of the 2001 Particle Accelerator Conference, Chicago, 2001, p. 1859.

[7] W. Bruns, GDFIDL, http://www.GDFIDL.de.

[8] B. Podobedov and S. Krinsky, Proceedings of the 2006 EPAC-2006, Edinburgh, 2006, p. 2973.

[9] A. Chao, S. Heifets, and B. Zotter, Phys. Rev. ST Accel. Beams 5, 111001 (2002).

[10] T.F. Günzel, Phys. Rev. ST Accel. Beams 9, 114402 (2006).

[11] R. Nagaoka, Proceedings of the 2001 Particle Accelerator Conference, Chicago, 2001, p. 3531.

[12] A. Burov and V. Danilov, Phys. Rev. Lett. 82, 2286 (1999).

[13] A. W. Chao, Physics of Collective Beam Instabilities in High Energy Accelerators (John Wiley \& Sons, Inc., New York, 1993), p. 192.

[14] L. Emery, G. Decker, and J. Galayda, Proceedings of the 2001 Particle Accelerator Conference, Chicago, 2001, p. 1823.

[15] V. Kiselev and V. Smaluk, Nucl. Instrum. Methods Phys. Res., Sect. A 525, 433 (2004).

[16] T. Perron, Ph.D. thesis, Université Joseph Fourier, Grenoble, France, 2005. 
[17] G. Nassibian and F. Sacherer, Nucl. Instrum. Methods 159, 21 (1979).

[18] K. Yokoya, Part. Accel. 41, 221 (1993).

[19] R. Nagaoka, Proceedings of EPAC 2004, Lucerne, Switzerland, 2004, p. 2041.

[20] NSLS-II CDR, 2006.

[21] Waveguide Handbook, edited by N. Marcuvitz (Dover, New York, 1965).

[22] A. Blednykh, Nucl. Instrum. Methods Phys. Res., Sect. A 565, 380 (2006).

[23] S. Zhang and Y. Shen, IEEE Trans. Microwave Theory Tech. 43, 1 (1995).

[24] Handbook of Accelerator Physics and Engineering, edited by A. W. Chao and M. Tigner (World Scientific, Singapore, 1998).

[25] P. Tenenbaum et al., Phys. Rev. ST Accel. Beams 10, 034401 (2007).

[26] K. Bane (private communication).

[27] Y.-H. Chin, CERN SL/94-02 (AP).

[28] Wolfram Research, http://www.wolfram.com.

[29] R. L. Gluckstern, J. van Zeijts, and B. Zotter, Phys. Rev. E 47, 656 (1993).

[30] O. Napoly, Y.-H. Chin, and B. Zotter, Nucl. Instrum. Methods Phys. Res., Sect. A 334, 255 (1993).

[31] I. Zagorodnov and T. Weiland, Phys. Rev. ST Accel. Beams 8, 042001 (2005). 